Research Article

\title{
Bayesian Uncertainty Identification of Model Parameters for the Jointed Structures with Nonlinearity
}

\author{
Zhanpeng Shen $\mathbb{D},{ }^{1,2}$ Xinen Liu, ${ }^{2}$ Chaoping Zang $\mathbb{D},{ }^{1}$ and Shaoquan $\mathrm{Hu}^{2}$ \\ ${ }^{1}$ Jiangsu Province Key Laboratory of Aerospace Power System, College of Energy and Power Engineering, \\ Nanjing University of Aeronautics and Astronautics, Nanjing 210016, China \\ ${ }^{2}$ Institute of System Engineering, China Academy of Engineering Physics, Mianyang 621999, China
}

Correspondence should be addressed to Chaoping Zang; c.zang@nuaa.edu.cn

Received 2 September 2021; Accepted 1 November 2021; Published 22 November 2021

Academic Editor: Zeqi Lu

Copyright (c) 2021 Zhanpeng Shen et al. This is an open access article distributed under the Creative Commons Attribution License, which permits unrestricted use, distribution, and reproduction in any medium, provided the original work is properly cited.

\begin{abstract}
Jointed structures in engineering naturally perform with some of nonlinearity and uncertainty, which significantly affect the dynamic characteristics of the structural system. In this paper, the method of Bayesian uncertainty identification of model parameters for the jointed structures with local nonlinearity is proposed. Firstly, the nonlinear stiffness and damping of the joints under the random excitation are represented with functions of excitation magnitude in terms of the equivalent linearization. The process of uncertainty identification is separated from the representation of local nonlinearity. In this way, the dynamic behavior of the joints is penetratingly characterized instead of ascribing the nonlinearity to uncertainty. Secondly, a variable-expanded Bayesian (VEB) method is originally proposed to identify the mixed of aleatory and epistemic uncertainties of model parameters. Different from traditional Bayesian identification, the aleatory uncertainties of model parameters are identified as one of the most important parts rather than only measurement noise of output. Notablely, a series of intermediate variables are introduced to expand the parameter with aleatory uncertainty in order to overcome the difficulty of establishing the likelihood function. Moreover, a 3-DOF numerical example is illustrated with case studies to verify the proposed method. The influence of observed sample size and prior distribution selection on the identification results is tested. Furthermore, an engineering example of the jointed structure with rubber isolators is performed to show the practicability of the proposed method. It is indicated that the computational model updated with the accurately identified parameters with both nonlinearity and uncertainty has shown the excellent predictive capability.
\end{abstract}

\section{Introduction}

Engineering structures with joints usually behave with some of the nonlinear vibration characteristics. These nonlinear phenomena are usually concentrated in the local joints of the parts, such as bolt flanges, interference/clearance fit, cushion, and vibration isolator. It may lead to complex nonlinear responses such as natural frequency shift, phase distortion, frequency response jump, and other phenomena [1-3] with the increase of excitation levels. Though calculation of the direct nonlinear responses has been studied for many years, it is still in difficulty for dealing with engineering structures with a huge number of degrees of freedom (DOFs), especially under random excitations. The equivalent linearization to deal with nonlinearity is currently adopted because of the maturity and convenience to calculate the random vibration response [4,5]. Friis et al. [4] summarized three commonly used equivalent methods with numerical examples, including the energy balance method, the intensity balance method, and the direct linear fit in the least squares sense. Alibrandi and Mosalam [6] proposed three linear equivalence methods for reliability evaluation of random vibration structures, that is, Gaussian linear equivalent linearization, tail equivalent linearization, and tail probability equivalent linearization. Other researchers have also done many studies about equivalent linearization under random vibration $[7,8]$. However, the authors insist that the equivalent linearization model should address the nonlinear features such 
as the fact that the equivalent stiffness and damping are represented with functions of excitation magnitudes. Chen et al. [9], respectively, established the polynomial models for equivalent stiffness and equivalent damping varying with excitation magnitudes in the study of a rubber vibration isolator. The polynomial coefficients are analytically derived according to the sin-sweep vibration results in different test cases.

From another point of view, the model parameters inevitably show some of uncertainties no matter linear or nonlinear model to be built up $[10,11]$. For example, the modulus of some polymer materials like rubber could vary $20 \%$ among the same manufacture batch. In fact, the equivalent stiffness and damping of the component joints are also uncertain, because of the joint interface consisting of a large number of microconvex bodies with random sizes and positions. The uncertainties in model parameters may be characterized as either aleatory uncertainties, which are irreducible variabilities inherent in nature, or epistemic uncertainties, which are reducible uncertainties [11, 12] resulting from a lack of knowledge or intentional simplifications. As opposed to model parameter uncertainty, model form uncertainty $[13,14]$ is also epistemic and worthy of note, but it is not the focus of this paper. Model parameters are normally uncertain and therefore uncertainty also exists in the dynamic response. Moreover, it is usually much greater than measurement noise. As a result, the uncertainties as well as the optimal estimations for parameters should be identified in order to predict the uncertainty of the response.

Though there have been lots of literatures to study the nonlinear dynamic behaviors of the jointed structure such as the vibration isolator [15-17], the uncertainties of model parameters are not often identified. Kerschen et al. [3] have made a detailed summary of parameter identification methods for nonlinear structures and divided them into seven categories. Therein, structural model updating $[18,19]$ is more convenient than other methods and recommendable for the complex structure due to large number of DOFs in the finite element model. Moreover, among various model updating methods, Bayesian inference is a very popular probabilistic identification method, which has been widely used in both linear and nonlinear structures [20-27]. Compared with the deterministic method based on optimization, Bayesian method can more flexibly deal with modelling uncertainties and measurement noise.

However, most of the current Bayesian parameter identification methods focus on the optimal estimation, and the main uncertainty is considered to be the measurement noise. The probability distribution functions (PDFs) of the model parameters with aleatory uncertainty are scarcely obtained due to the high cost of repeated experiments to adequately describe the aleatory uncertainties, though statistical variance is identified to address uncertainty in some good way. The other difficulty is to establish the likelihood function since the uncertainty of experimental data comes from both the measurement noise and the uncertainty of parameters. It indicates that the experimental data of output cannot be generally assumed as the normal distributed and the likelihood function cannot be directly analytical expressed.

In this paper, a new VEB method is originally proposed to identify the PDFs of model parameters with the mixed aleatory and epistemic uncertainties based on the experimental data. A series of intermediate variables with the same prior distribution are introduced, and the model parameters with aleatory uncertainty are tactfully transformed in order to establish the new likelihood function. Combined with the equivalent linearization under different magnitudes of random excitations, the local nonlinearity is also characterized together with the uncertainties for jointed structures. Compared with traditional Bayesian identification, uncertainties of model parameters are identified based on repeated experimental data rather than only measurement noise of output. It is highlighted herein that the influences of parameters' uncertainties (e.g., elastic modulus of the rubbers in isolators) on structural responses are usually critical in engineering rather than measurement noise. Compared with interval analysis or other variance-based methods, more detailed information of the uncertainties could be achieved in terms of PDF and the identification results could be quickly updated with the additional useful experimental data. Moreover, the nonlinearity of the joints is separated from the uncertainty, leading to the fact that the dynamic behavior is penetratingly characterized instead of ascribing the nonlinearity to uncertainty. Thus, the computational model updated with this method has a high predictive capability for the jointed structures.

The paper is organized as follows. Section 2 provides a brief introduction to the local nonlinearity modelling of the jointed structures. Section 3 then introduces the Bayesian identification of the uncertainties for model parameters including the VEB method in detail. Section 4 studies a virtual 3-DOF spring mass system with 3 different cases to illustrate and verify the proposed method. Moreover, Section 5 provides an engineering example of the jointed structures with rubber isolators to show the practicability of the proposed method. Finally, Section 6 ends with our main conclusions.

\section{Local Nonlinearity Modelling of the Jointed Structures}

The jointed structures could be regarded as a combination of many components. Generalized spring-damping elements can be adopted to characterize the joints between the boundary and components or among different components. Some of elements are linear while some are nonlinear as shown in Figure 1. It depends on the mechanical behavior of the joints.

For the nonlinear system, the motion equation can be written as

$$
\mathbf{M y}+\mathbf{C} \dot{\mathbf{y}}+\mathbf{K y}+\mathbf{f}_{n l}(\mathbf{y}, \dot{\mathbf{y}})=\mathbf{x}(t),
$$

where $\mathbf{x}(t)$ denotes the excitation vector varying with time and $\mathbf{y}(t)$ denotes the displacement solution. $\mathbf{M}, \mathbf{C}$, and $\mathbf{K}$ are, respectively, the mass, damping, and stiffness matrices of the 


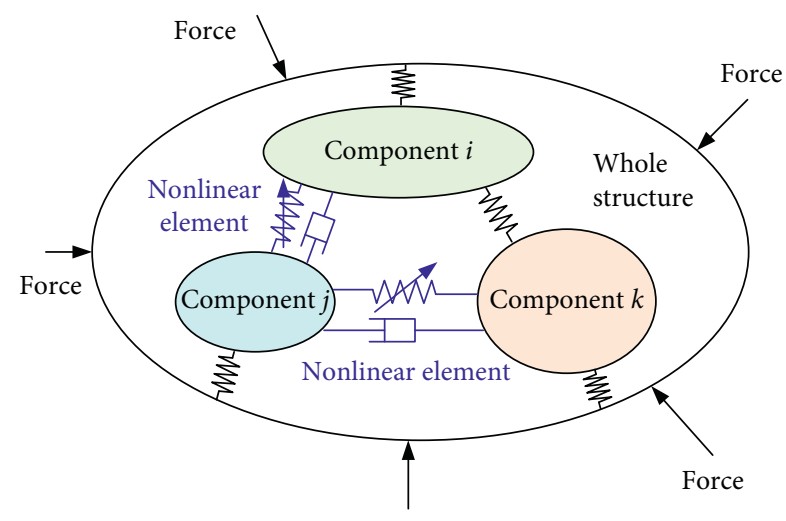

Vibration of ground

FIGURE 1: Illustration of the nonlinear spring-damping elements for the jointed structures.

system, and $\mathbf{f}_{n l}$ is the nonlinear term. For the weak nonlinearity, equivalent linearization is often adopted in engineering. The equivalent equation can be written as

$$
\mathbf{M} \ddot{\mathbf{y}}+\mathbf{C}_{e q} \dot{\mathbf{y}}+\mathbf{K}_{e q} \mathbf{y}=\mathbf{x}(t),
$$

where $\mathbf{C}_{e q}$ and $\mathbf{K}_{e q}$ are equivalent damping and equivalent stiffness matrices, respectively. Compared with $\mathbf{C}$ and $\mathbf{K}$, only a few elements of $\mathbf{C}_{e q}$ and $\mathbf{K}_{e q}$ change because the nonlinearity of the jointed structures only appears in a few local positions.

Due to random excitation input, the nonlinearity of the jointed structures is averaged or linearized in a sense [28]. The frequency response function (FRF) of the nonlinear system under the random excitation is defined by (3). It appears to be linear in terms of its shape with eigenfrequency drifting as shown in Figure 2(a):

$$
H_{n r}(\omega)=\frac{S_{y x}(\omega)}{S_{x x}(\omega)},
$$

where $S_{y x}(\omega)$ and $S_{x x}(\omega)$ are the cross- and auto-spectrum densities, respectively. It should be noted that the FRF of the nonlinear system under the random excitation (i.e., $H_{n r}(\omega)$ ) shows clear distinction from that under the sin-sweep excitation. It mainly depends on the input power spectral density (i.e., the vibration magnitude), and it approaches the linear FRF as the power spectral density approaches zero.

Taking modal parameters (i.e., natural frequency and damping ratio) from the FRFs with different vibration magnitudes as the target, the equivalent stiffness and damping of the nonlinear elements could be obtained, respectively, as shown in the following:

$$
\begin{aligned}
& \mathbf{k}_{e q}(A)=\left[k_{e q 1}(A), k_{e q 2}(A), \ldots, k_{e q, s}(A)\right]^{T}, \\
& \mathbf{c}_{e q}(A)=\left[c_{e q 1}(A), c_{e q 2}(A), \ldots, c_{e q, s}(A)\right]^{T},
\end{aligned}
$$

where $s$ denotes the number of nonlinear elements and $A$ denotes the magnitude of the random excitation. According to the FRF of a nonlinear system with a signal DOF under different magnitudes of random excitation as shown in Figure 2(a), the equivalent stiffness and damping of the nonlinear spring-damping element are shown in Figure 2(b), respectively. It should be noted that the FRF in Figure 2 appears undistorted and seems like a linear FRF just with frequency drift. The reason is not soft nonlinearity but is the fact that the randomness of the amplitude and phase of the excitation signal creates a "linearized" or "averaged" FRF.

\section{Bayesian Identification of the Model Parameter Uncertainties}

Compared with the determinate parameter identification, Bayesian identification provides not only the optimal estimations of the model parameters but also the probability distribution to describe their uncertainties. Generally, the structural response of interest (e.g., natural frequency or modal damping ratio) can be expressed in terms of components as follows:

$$
z_{i}=z_{i}(\mathbf{x} ; \boldsymbol{\theta}, \boldsymbol{\alpha}), \quad i=1,2, \ldots, q,
$$

where $q$ is the number of output responses, $\mathbf{x}$ is the controllable and determinate input vector (e.g., the random vibration magnitude), $\boldsymbol{\theta}$ denotes the aleatory uncertain parameter set, and $\boldsymbol{\alpha}$ denotes the epistemic uncertain parameter set.

It is noted that $\boldsymbol{\theta}$ has observable randomness and its uncertainty is inherent and irreducible. However, $\boldsymbol{\alpha}$ has an unknown but unique truth value, whose uncertainty can be reduced with the addition of useful information. These two types of parameters are independent with each other. In addition, the prior distributions of the aleatory uncertain parameter could be expressed as $\pi_{\boldsymbol{\theta}}\left(\boldsymbol{\theta} \mid \boldsymbol{\alpha}_{1}\right)$, where the hyperparameter set $\boldsymbol{\alpha}_{1}$ has also epistemic uncertainty and does not appear in the model.

Ignoring the model form error, experimental error is assumed to follow the normal distribution with zero mean, and then the experimental observation of each output can be expressed as

$$
\left\{\begin{array}{l}
\widetilde{z}_{i}=z_{i}(\mathbf{x} ; \boldsymbol{\theta}, \boldsymbol{\alpha})+\varepsilon_{i}, \\
\varepsilon_{i} \sim N\left(0, \sigma_{i}^{2}\right),
\end{array} \quad i=1,2, \ldots, q .\right.
$$

Denoting the $n$ groups of test data set as $\mathbf{D}=\left\{\mathbf{x}_{k}, \widetilde{z}_{k}\right\}, k=1,2, \ldots, n$, there are unknown values of $\theta_{k}$ and $\varepsilon_{k}$ corresponding to each set of test data $\left(\mathbf{x}_{k}, \mathbf{z}_{k}\right)$; that is,

$$
\widetilde{z}_{k i}=z_{i}\left(\mathbf{x}_{k} ; \boldsymbol{\theta}_{k}, \boldsymbol{\alpha}\right)+\varepsilon_{k i}, \quad i=1,2, \ldots, q, k=1,2, \ldots, n .
$$

\footnotetext{
3.1. Brief Introduction of Traditional Bayesian Identification of Model Parameters. Traditional Bayesian parameter identification, namely, Bayesian model updating, deals with only the epistemic uncertain variables. Measurement error is assumed to be aleatory uncertainty, and each of the model parameters is assumed to have an unknown unique true value. The belief in the true value is represented with probability distribution in Bayesian method.
} 


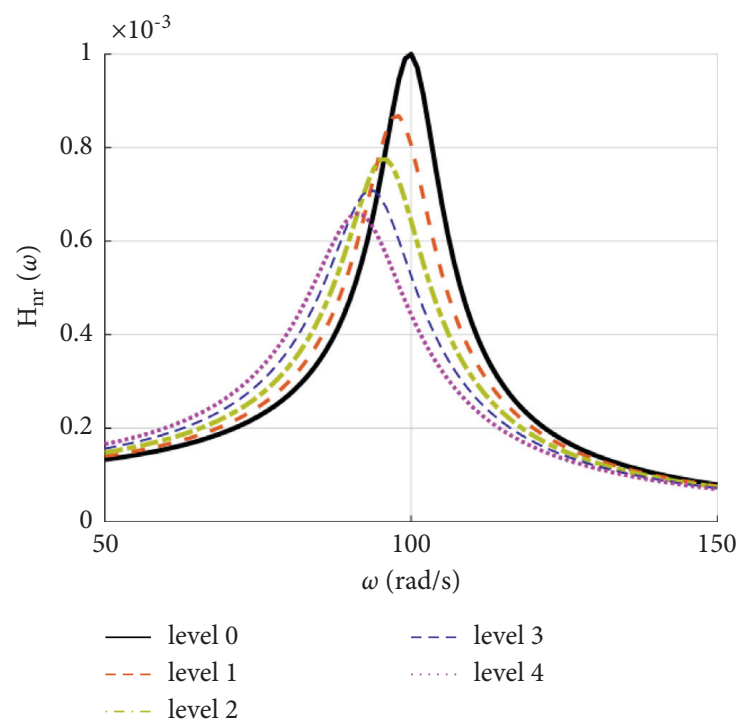

(a)
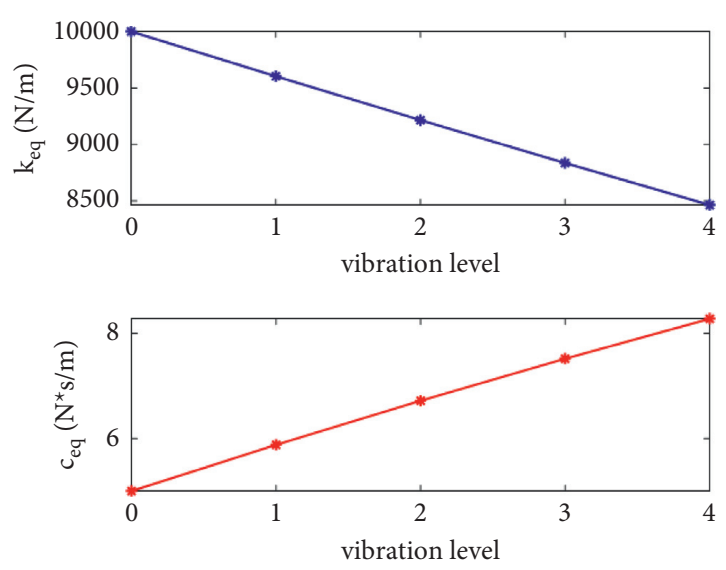

FIgURE 2: Equivalent linearization of nonlinear spring-damping element with different vibration magnitudes. (a) FRFs and (b) equivalent stiffness and equivalent damping.

Ignoring the aleatory uncertain variables $\boldsymbol{\theta}$ in (6) (8), the posterior distribution of parameters $\boldsymbol{\alpha}$ is obtained according to test data set $\mathbf{D}$ by using the Bayesian formula:

$$
p(\mathbf{\alpha} \mid \mathbf{D})=\frac{L(\mathbf{D} \mid \mathbf{\alpha}) \pi_{\alpha}(\boldsymbol{\alpha})}{\int L(\mathbf{D} \mid \mathbf{\alpha}) \pi_{\alpha}(\boldsymbol{\alpha}) \mathrm{d} \boldsymbol{\alpha}} \propto L(\mathbf{D} \mid \mathbf{\alpha}) \pi_{\alpha}(\boldsymbol{\alpha}),
$$

where $\pi_{\alpha}(\alpha)$ is the prior PDF of $\boldsymbol{\alpha}$ and $L(\bullet)$ is the likelihood function. The denominator in (9) is an integral constant ensuring that the posterior PDF integrates to 1 .

Assuming that the test data of output are independent identically distributed (i.i.d) samples and test error follows normal distribution, the likelihood function for a single output can be constructed as

$$
\begin{aligned}
L_{i}\left(\mathbf{D} \mid \boldsymbol{\alpha}, \sigma_{i}\right) & =\prod_{k=1}^{n} \frac{1}{\sigma_{i} \sqrt{2 \pi}} \exp \left(-\frac{1}{2 \sigma_{i}^{2}}\left(\widetilde{z}_{k i}-z_{i}\left(\mathbf{x}_{k} ; \boldsymbol{\alpha}\right)\right)^{2}\right) \\
& =\frac{1}{(2 \pi)^{n / 2} \sigma_{i}^{n}} \exp \left(-\frac{1}{2 \sigma_{i}^{2}} \sum_{k=1}^{n}\left(\widetilde{z}_{k i}-z_{i}\left(\mathbf{x}_{k} ; \boldsymbol{\alpha}\right)\right)^{2}\right)
\end{aligned}
$$

If Bayesian identification is performed simultaneously using multivariate outputs, one definition of the likelihood function is shown in the following:

$$
L(\mathbf{D} \mid \mathbf{\alpha}, \boldsymbol{\sigma})=\prod_{i=1}^{q} \eta_{i} L_{i}\left(\mathbf{D} \mid \mathbf{\alpha}, \sigma_{i}\right),
$$

where $\eta_{i}$ is the weighted coefficient and $\boldsymbol{\sigma}=\left[\sigma_{1}, \sigma_{2}, \ldots, \sigma_{q}\right]$ is the test error vector.

3.2. Basic Theory of VEB Identification. Due to aleatory uncertainty in model parameters, uncertainties in output data do not just come from the measurement error. The whole observed data cannot be generally assumed as the normal distributed. Moreover, if the model outputs do not linearly vary with aleatory uncertain parameters or these parameters are not conjugate distributed (e.g., normally distributed), the likelihood function cannot be analytically expressed directly. Furthermore, the calculation amount of the likelihood function value in terms of numerical expression is usually too huge to implement in Bayesian method. As a result, the traditional Bayesian identification encounters generally serious difficulty when the aleatory uncertainty in model parameters cannot be neglected.

Assuming the $n$ sets of test data are independent with each other, a series of intermediate variables, namely, $\left[\boldsymbol{\theta}_{1}, \boldsymbol{\theta}_{2}, \ldots, \boldsymbol{\theta}_{n}\right]$, with the same prior distributions are introduced to temporarily replace the aleatory uncertain parameters $\boldsymbol{\theta}$. Each vector $\boldsymbol{\theta}_{k}$ of the intermediate variables corresponds to every sample set of the aleatory uncertain parameters. Each intermediate variable is regarded as the denotation of the true value of the corresponding test sample with only epistemic uncertainty. Thus, each set of the output test data $\widetilde{z}_{k}$ is randomly distributed around $\mathbf{z}_{k}\left(\mathbf{x} ; \boldsymbol{\theta}_{k}, \boldsymbol{\alpha}\right)$ and the test error could be assumed as a normal random variable 
with zero mean. Hence, the likelihood function could be constructed as

$$
\begin{aligned}
& L_{i}\left(\mathbf{D} \mid \boldsymbol{\theta}_{k}: k=1 \ldots n, \boldsymbol{\alpha}, \sigma_{i}\right)=\frac{1}{(2 \pi)^{n / 2} \sigma_{i}^{n}} \exp \left(-\frac{1}{2 \sigma_{i}^{2}} \sum_{k=1}^{n}\left(\widetilde{z}_{k i}-z_{i}\left(\mathbf{x}_{k} ; \boldsymbol{\theta}_{k}, \boldsymbol{\alpha}\right)\right)^{2}\right), \\
& L\left(\mathbf{D} \mid \boldsymbol{\theta}_{k}: k=1 \ldots n, \boldsymbol{\alpha}, \boldsymbol{\sigma}\right)=\prod_{i=1}^{q} \eta_{i} L_{i}\left(\mathbf{D} \mid \boldsymbol{\theta}_{k}: k=1 \ldots n, \boldsymbol{\alpha}, \sigma_{i}\right),
\end{aligned}
$$

where the test data set $\mathbf{D}$ is shown in (8).
Then, according to Bayesian theorem, the posterior joint PDF for all parameters can be expressed as

$$
p\left(\boldsymbol{\theta}_{k}: k=1 \ldots n, \boldsymbol{\alpha}, \boldsymbol{\alpha}_{1}, \boldsymbol{\sigma} \mid \mathbf{D}\right) \propto L\left(\mathbf{D} \mid \boldsymbol{\theta}_{k}: k=1 \ldots n, \boldsymbol{\alpha}, \boldsymbol{\sigma}\right) \prod_{k=1}^{n} \pi_{\theta}\left(\boldsymbol{\theta}_{k} \mid \boldsymbol{\alpha}_{1}\right) \pi_{\alpha}(\boldsymbol{\alpha}) \pi_{\alpha_{1}}\left(\boldsymbol{\alpha}_{1}\right) \pi_{\sigma}(\boldsymbol{\sigma})
$$

where $\pi_{\theta}\left(\theta_{k} \mid \alpha_{1}\right), \pi_{\alpha}(\alpha)$, and $\pi_{\sigma}(\sigma)$, respectively, denote the prior PDFs for intermediate variable $\boldsymbol{\theta}_{k}$, epistemic uncertain parameter $\boldsymbol{\alpha}$ and the experimental standard deviation $\boldsymbol{\sigma}$. $\pi_{\alpha_{1}}\left(\alpha_{1}\right)$ denotes the prior PDF for the hyperparameter $\boldsymbol{\alpha}_{1}$ addressing the distribution of $\boldsymbol{\theta}_{k}$.

Integrating the intermediate variables, the posterior joint $\mathrm{PDF}$ is rewritten as

$$
p\left(\boldsymbol{\alpha}, \boldsymbol{\alpha}_{1}, \boldsymbol{\sigma} \mid \mathbf{D}\right)=\int_{\theta_{k}} p\left(\boldsymbol{\theta}_{k}: k=1 \ldots q, \boldsymbol{\alpha}, \boldsymbol{\alpha}_{1}, \boldsymbol{\sigma} \mid \mathbf{D}\right) \prod_{k=1}^{q} \mathrm{~d} \boldsymbol{\theta}_{k} .
$$

By marginalizing equation (14), the posterior PDF for any epistemic uncertain parameter can be obtained.

The posterior PDFs for aleatory uncertain parameters can be acquired by integrating the hyperparameters as shown in (15). Essentially, the result is weighted average by the posterior probability of the hyperparameters:

$$
\begin{aligned}
p(\boldsymbol{\theta} \mid \mathbf{D}) & =\int_{\alpha_{1}} \pi_{\theta}\left(\boldsymbol{\theta} \mid \boldsymbol{\alpha}_{1}\right) p\left(\boldsymbol{\alpha}_{1} \mid \mathbf{D}\right) d \boldsymbol{\alpha}_{1} \\
& =\int_{\alpha, \alpha_{1}, \sigma} \pi_{\theta}\left(\boldsymbol{\theta} \mid \boldsymbol{\alpha}_{1}\right) p\left(\boldsymbol{\alpha}, \boldsymbol{\alpha}_{1}, \boldsymbol{\sigma} \mid \mathbf{D}\right) d \boldsymbol{\alpha} d \boldsymbol{\alpha}_{1} d \boldsymbol{\sigma} .
\end{aligned}
$$

3.3. Algorithm Implementation of VEB Identification. Due to the mathematical difficulties of the high-dimensional integration, Bayesian posterior distribution is generally estimated by an effective stochastic simulation method called Markov chain Monte Carlo (MCMC) [29-31] instead of the direct integration. It generates a long sequence of samples from the complex shaped unknown posterior distributions. Metropolis-Hasting algorithm is a classical useful algorithm to generate the MCMC samples.

Moreover, further management needs to be performed because of the asymptotic convergence and autocorrelation for the MCMC samples in sequence. The head part of the sample sequence which has not been convergent is removed. The whole sequence is diluted by extracting a sample at a certain interval to ensure that the autocorrelation can be ignored. Then, the useful MCMC samples are obtained to characterize the posterior distribution of each variable.

The sample data are usually arranged into a matrix, where each column corresponds to each parameter to be identified. It is convenient to obtain the marginal distribution of each parameter or study the correlation between parameters base on the sample matrix. It is also very easy to get the posterior joint PDF or marginal PDFs for $\left\{\boldsymbol{\alpha}, \boldsymbol{\alpha}_{1}, \boldsymbol{\sigma}\right\}$ by sample matrix instead of (14). The corresponding columns of intermediate variables are just discarded from the full matrix, and the remaining sample data are employed to address the joint PDF.

In order to acquire the accurate posterior distribution, the sample sequence should be long enough after head removing and dilution. In other words, a huge amount of MCMC samples is required to calculate. In general, the computational efficiency depends on the calculation cost of outputs represented by (6), which are used for likelihood function calculation. Unfortunately, structural outputs are usually expensively computed by solving the finite element model in engineering. Thus, efficient meta-model with high accuracy is required to build up, such as polynomial response surface, Kriging model, artificial neural network, and polynomial chaotic expansion.

The flowchart is shown in Figure 3 to illustrate the implementation of VEB identification. First of all, the uncertain parameters to be identified should be clearly classified according to their attributes. Then, nonlinear modelling, probabilistic modelling. and modal testing are carried out. The process of MCMC is performed to obtain the posterior samples of all parameters. Consequently, the samples should be verified to satisfy the requirement of asymptotic convergence and autocorrelation. Finally, the posterior PDF of the parameters could be gained from the 


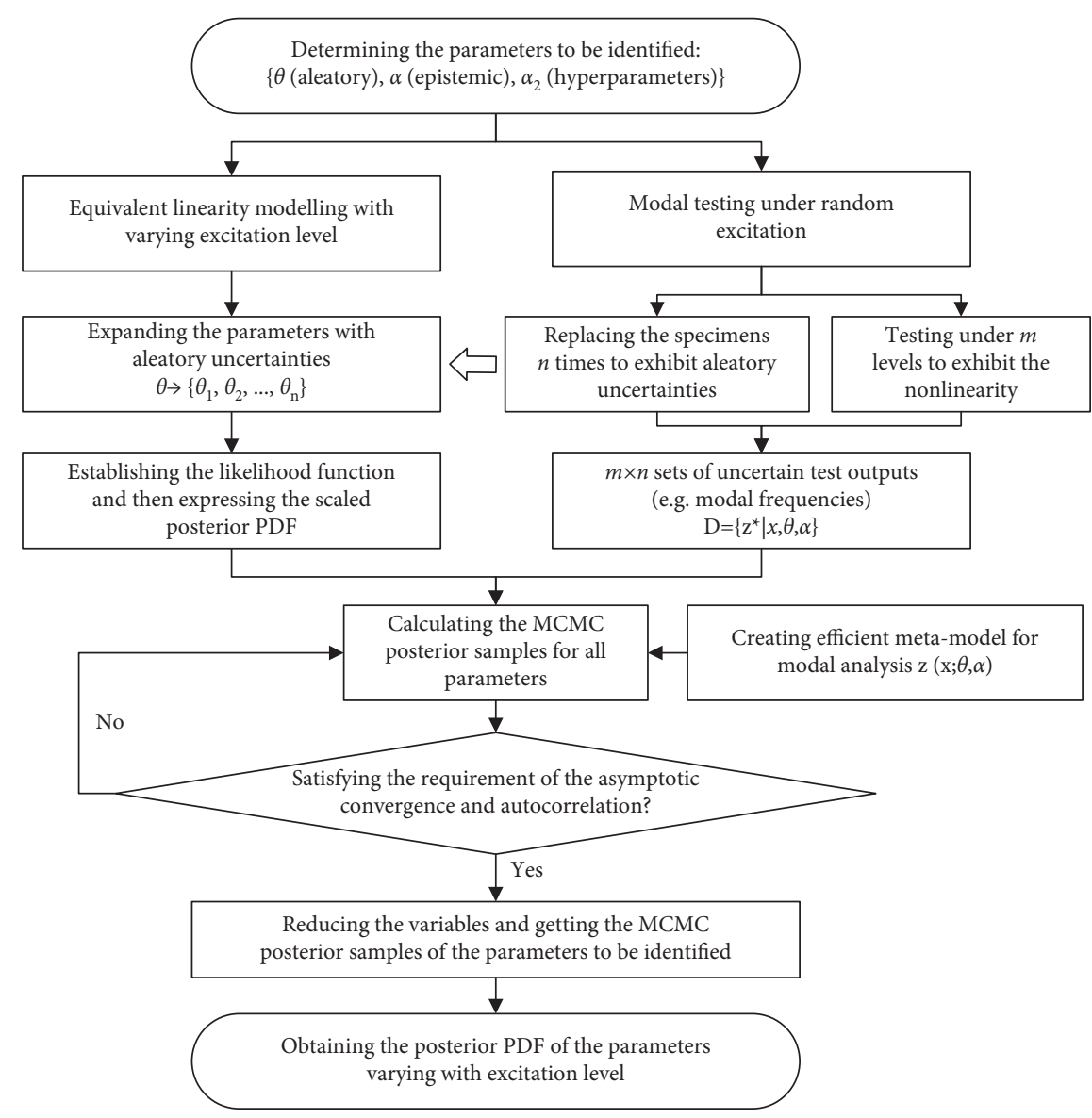

Figure 3: The flowchart of the VEB identification method.

reduced MCMC samples to represent both the nonlinearity and uncertainties.

Frankly speaking, VEB identification costs more computational amount than traditional Bayesian method, because the variables to be identified are expanded and the convergence rate of MCMC slows down. Denoting the numbers of aleatory and epistemic uncertain parameters by $r$ and $s$, respectively, $n r+s$ variables (including intermediate variables) need to generate samples in MCMC processing, where $n$ denotes the number of test groups for aleatory uncertain parameters. Thus, the variables would expand more serious as the number of test groups increases. Moreover, it needs longer time and more computational amount to get convergent MCMC samples using. However, the computational cost of VEB identification is generally acceptable and controllable. On one hand, the number $n$ would not be too large due to expensive experimental cost. On the other hand, the advantage of Bayesian inference method lies in making full use of prior information and less test data to obtain accurate estimates. It prefers identifying the parameters and updating the results again and again according to the current small amount of new test data, rather than waiting for allocating a large number of test data and identifying them in one time.

3.4. Output Prediction. For structural analysis in engineering, our purpose of parameters identification is usually predicting the output accurately under different inputs. The advantage of uncertainty identification in this paper is that probability distribution of predictive output could also be addressed for further study.

Ignoring the experimental errors in prediction, the predictive output vector is expressed as $\mathbf{z} \prime=\mathbf{z} \prime(\mathbf{x} \prime, \theta, \alpha)$ and its probability distribution is determined by the distributions of parameters $\boldsymbol{\theta}$ and $\boldsymbol{\alpha}$. Thus, the PDF for $\mathbf{z}^{\prime}$ could be addressed with its samples, which could be calculated by (6) according to the posterior MCMC samples for $\boldsymbol{\theta}$ and $\boldsymbol{\alpha}$.

With consideration of experimental errors in prediction, the predictive output vector is expressed as

$$
\mathbf{z}^{*}=\mathbf{z}^{\prime}\left(\mathbf{x}^{\prime}, \boldsymbol{\theta}, \boldsymbol{\alpha}\right)+\boldsymbol{\varepsilon} .
$$

The conditional PDF of the predictive outputs could be written as 


$$
p\left(z_{i}^{*} \mid \boldsymbol{\theta}, \boldsymbol{\alpha}, \sigma_{i}\right)=\frac{1}{(2 \pi)^{1 / 2} \sigma_{i}} \exp \left(-\frac{1}{2 \sigma_{i}^{2}}\left(z_{i}^{*}-z_{i}^{\prime}(\mathbf{x} \prime, \boldsymbol{\theta}, \boldsymbol{\alpha})\right)^{2}\right), \quad i=1,2, \ldots, q .
$$

From Bayesian theory, the posterior PDF of predictive outputs is expressed as

$$
p\left(z_{i}^{\prime} \mid \mathbf{D}\right)=\int_{\theta, \alpha, \alpha_{1}, \sigma} p\left(z_{i}^{\prime} \mid \boldsymbol{\theta}, \boldsymbol{\alpha}, \sigma_{i}\right) p\left(\boldsymbol{\alpha}, \boldsymbol{\alpha}_{1}, \sigma_{i} \mid \mathbf{D}\right) \pi_{\theta}\left(\boldsymbol{\theta} \mid \boldsymbol{\alpha}_{1}\right) d \boldsymbol{\theta} \mathrm{d} \boldsymbol{\alpha} \mathrm{d} \boldsymbol{\alpha}_{1} \mathrm{~d} \sigma_{i}, \quad i=1,2, \ldots, q
$$

\section{Numerical Examples}

4.1. Example Statement. A 3-DOF system with 4 linear springs and a nonlinear spring is oscillated under base excitation as shown in Figure 4. The virtual modal test of the system is accomplished, respectively, under random excitation with 6 acceleration levels, including $0.1 \mathrm{~g}, 1 \mathrm{~g}, 2 \mathrm{~g}, 4 \mathrm{~g}$, $6 \mathrm{~g}$, and $8 \mathrm{~g}$. Since the stiffness of the nonlinear spring $k_{4}$ varies with the vibration magnitudes, the FRF shifts as the excitation acceleration increases as shown in Figure 5.

In order to verify the effectiveness and practicability of the proposed method, three cases were studied as shown in Table 1 . The mass of each block is $0.1 \mathrm{~kg}$, while stiffness parameters $k_{1}$ and $k_{3}$ are equal to $1000 \mathrm{~N} / \mathrm{m}$ and $2000 \mathrm{~N} / \mathrm{m}$, respectively, for all cases. The outputs are 3 natural frequencies, while controllable input variable is the acceleration magnitude of base excitation. The example focuses on the identification of the other spring stiffness parameters, that is, $k_{2}, k_{4}$, and $k_{5}$.

In case 1 , the nonlinear stiffness $k_{4}$ is modelled with the quadratic polynomial, and the coefficients $\{a, b, c\}$ need to be identified. The traditional Bayesian identification is adopted with the consideration of measurement noise, and the uncertainties of model parameter cannot be identified. In case 2 , linear stiffness $k_{2}$ and $k_{5}$ with aleatory uncertainties are identified using VEB method. Symmetrical and asymmetrical PDFs are set for $k_{2}$ and $k_{5}$, respectively, that is, normal and gamma distributions. In case 3 , both nonlinear stiffness $k_{4}$ and linear stiffness $k_{2}$ and $k_{5}$ are identified together with their uncertainty using VEB method. Specifically, the constant term $c$ of the polynomial model for nonlinear stiffness $k_{4}$ is assumed as an aleatory uncertain parameter with normal distribution.

The natural frequencies from virtual modal test are generated from (19), where $\tilde{f}_{i}$ and $f_{i}$ denote $i$ th experimental and computational frequency and $x$ denotes the base excitation magnitude. The measurement noise $\varepsilon$ is normally distributed with zero mean and $0.02 \mathrm{~Hz}$ standard deviation:

$$
\tilde{f}_{i}=f_{i}\left(x ; k_{2}, k_{4}, k_{5}\right)+\varepsilon, \quad i=1,2,3 .
$$

4.2. Case Study 1: Nonlinear Stiffness Identification with consideration of Measurement Noise. The quadratic polynomial model for the stiffness of nonlinear spring verse excitation magnitude is established as

$$
k_{4}=a x^{2}+b x+c .
$$

The parameter set to be identified is $\alpha=\{a, b, c, \sigma\}$ in this case study, where $\sigma$ is the standard deviation of the test error. Traditional Bayesian identification is employed to obtain the posterior PDFs for $\{a, b, c, \sigma\}$ according to virtual experimental data at 6 excitation levels as shown in Figure 6. In fact, the second natural frequency is useless for nonlinearity identification, because the change is not obvious among different vibration levels. The uniform PDF in a wide range is taken as the prior distribution of the parameters.

The optimal identification values of the parameters are listed and compared with the true values in Table 2. The posterior PDFs of the parameters are shown in Figure 7. The sequence of MCMC samples for parameter $a$ are shown in Figure 8 as an example to demonstrate that the convergence and autocorrelation are satisfied.

It is indicated that the Bayesian identification results are very close to corresponding true values. Moreover, the posterior PDF becomes more and more sharp and the optimal estimation is generally closer to the true value as the amount of experimental data increases as shown in Table 2 and Figure 7. However, nothing is obtained for the aleatory uncertainty of model parameters. The epistemic uncertainties described in Figure 7 just come from the measurement noise and the identification error.

4.3. Case Study 2: Linear Stiffness Identification Together with Its Aleatory Uncertainties. Ignoring the nonlinear spring $k_{4}$, the linear model parameters, that is, spring stiffness $k_{2}$ and $k_{5}$, are set as the normal and gamma distribution, respectively, as shown in the following:

$$
\begin{aligned}
k_{2} & \sim N\left(\mu_{2}, \sigma_{2}^{2}\right), \\
k_{5} & =1000+\delta, \\
\delta & \sim \Gamma(d, e) .
\end{aligned}
$$

Virtual experimental frequency samples and the corresponding samples of spring stiffness are scattered as shown in Figure 9. In order to study the effect of the experimental data amount, 3, 5, and 10 sets of experimental samples are provided for VEB identification stage by stage. Because $1^{\text {st }}$ frequency is barely affected by $k_{2}$ and $k_{5}$, the experimental samples of $2^{\text {nd }}$ and $3^{\text {rd }}$ frequencies are utilized for VEB identification. In this case study, the aleatory uncertain parameter and the hyperparameter sets are $\theta=\left\{k_{2}, k_{5}\right\}$ and 


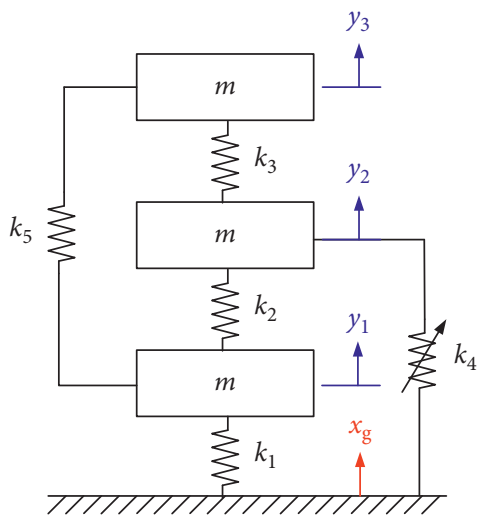

FIgURE 4: A 3-DOF oscillating system with linear and nonlinear springs.

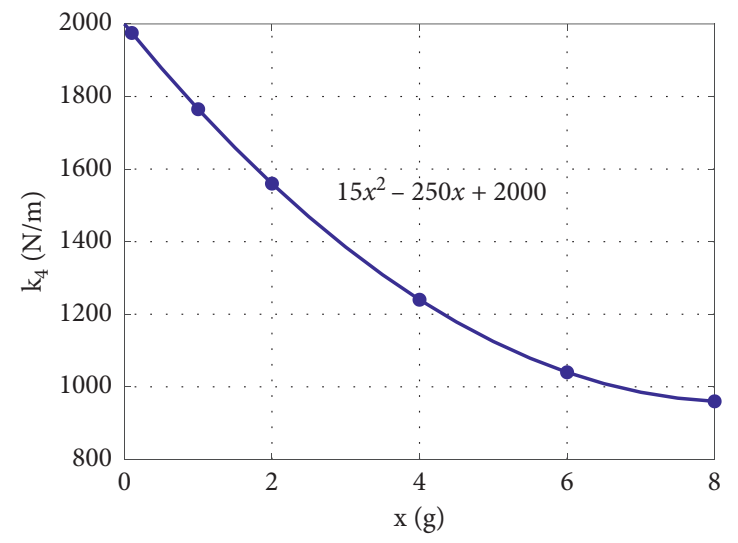

(a)
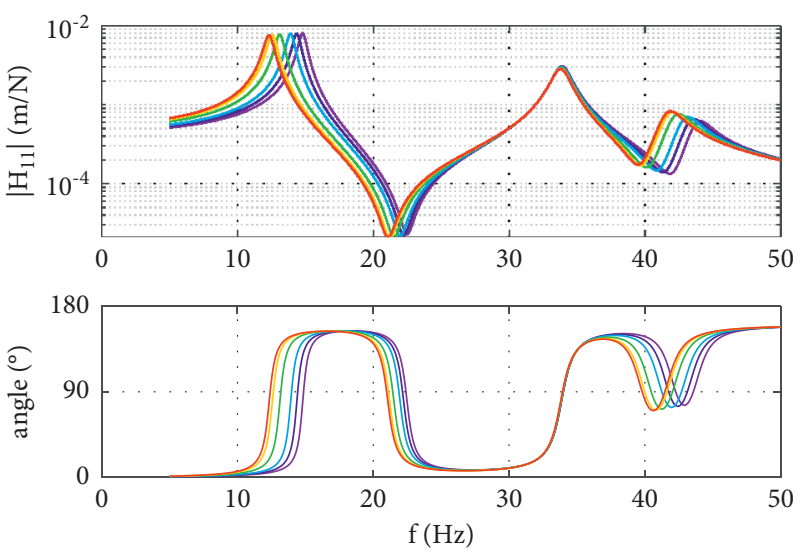

-0.
-1
-2

4

$-8$

(b)

FIgURE 5: Nonlinear feature of the 3-DOF system: (a) spring stiffness $k_{4}$ and (b) FRF vary with excitation magnitude.

TABLE 1: Comparison of the example cases.

\begin{tabular}{|c|c|c|c|c|}
\hline Item & & Case 1 & Case 2 & Case 3 \\
\hline Nonlinear/linear & & Nonlinear & Linear & Nonlinear \\
\hline Uncertainty & & Epistemic & Aleatory & Epistemic and aleatory \\
\hline \multirow{5}{*}{ Parameters $(\mathrm{N} / \mathrm{m})$} & $k_{1}$ & 1000 & 1000 & 1000 \\
\hline & $k_{2}$ & 2000 & Normal dist. & Normal dist. \\
\hline & $k_{3}$ & 2000 & 2000 & 2000 \\
\hline & $k_{4}$ & $a x^{2}+b x+c$ & 2000 & $\begin{array}{c}a x^{2}+b x+c \\
c \sim \text { normal dist. }\end{array}$ \\
\hline & $k_{5}$ & 1000 & Gamma dist. & Gamma dist. \\
\hline
\end{tabular}

$\alpha_{2}=\left\{\mu_{2}, \sigma_{2}, d, e\right\}$, respectively, while epistemic uncertain parameter set is empty.

As mentioned above, the advantage of Bayesian parameter identification lies in the full use of prior information, which can greatly reduce the demand for experimental sample size. Three forms of priors for hyperparameters are provided including uniform distribution, flat distribution, and sharp distribution, together with the comparison of their posterior PDFs, as shown in Figure 10. Similarly, the comparison for the aleatory uncertain parameters is shown in Figure 11. Identification errors are given in Table 3. 10 sets of experimental samples are provided for VEB identification herein.

The effect of different test sample sizes on identification results is also studied with flat prior distribution for the hyperparameters. The comparison is shown in Figure 12 and Table 3.

As the form of prior PDFs for the hyperparameters changes from "flat" to "sharp" ("uniform" is the flattest one), 


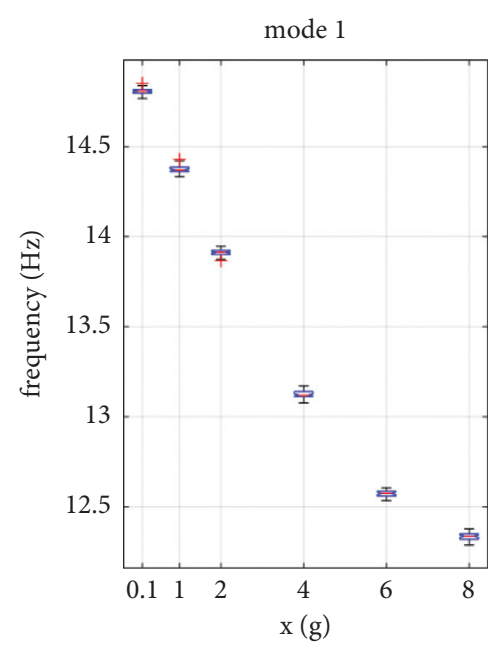

(a)

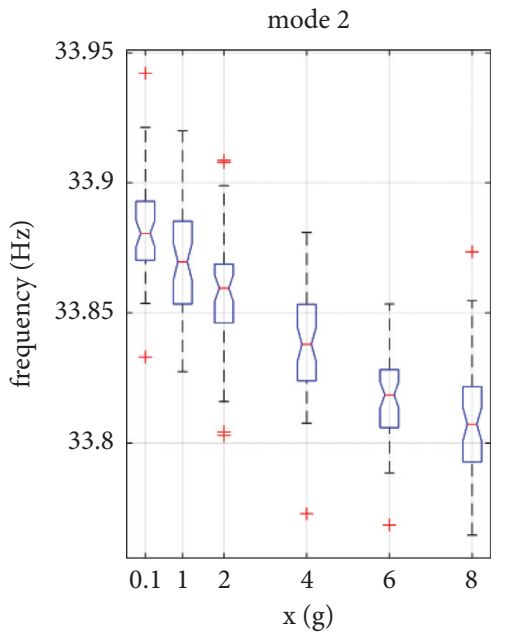

(b)

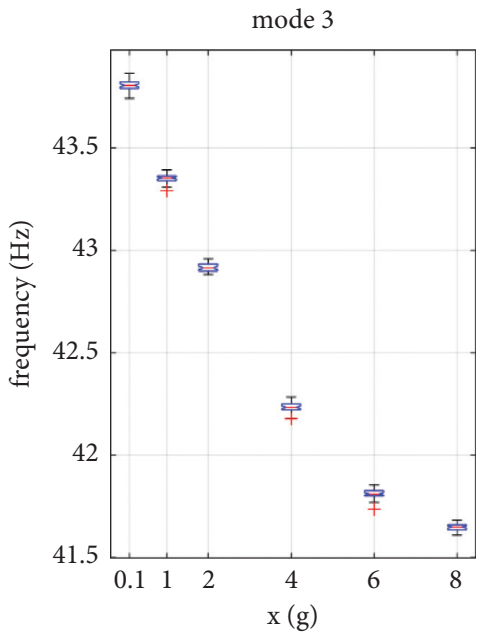

(c)

FIgURE 6: Boxplots of 50 sets of virtual experimental natural frequencies.

Table 2: Parameter identification results in case study 1.

\begin{tabular}{|c|c|c|c|c|c|c|c|}
\hline \multirow{3}{*}{$\begin{array}{l}\text { Parameter } \\
a\end{array}$} & \multirow{3}{*}{$\begin{array}{c}\text { True value } \\
15\end{array}$} & \multicolumn{6}{|c|}{ Identification value } \\
\hline & & \multicolumn{2}{|c|}{$n=1$} & \multicolumn{2}{|c|}{$n=5$} & \multicolumn{2}{|c|}{$n=50$} \\
\hline & & 15.252 & $1.68 \%$ & 15.146 & $0.97 \%$ & 14.984 & $-0.11 \%$ \\
\hline$b$ & -250 & -252.70 & $1.08 \%$ & -251.69 & $0.68 \%$ & -249.81 & $-0.08 \%$ \\
\hline$c$ & 2000 & 2002.9 & $0.15 \%$ & 2002.7 & $0.14 \%$ & 1999.7 & $-0.02 \%$ \\
\hline$\sigma$ & 0.02 & 0.01905 & $-4.77 \%$ & 0.02004 & $0.20 \%$ & 0.02054 & $2.68 \%$ \\
\hline
\end{tabular}

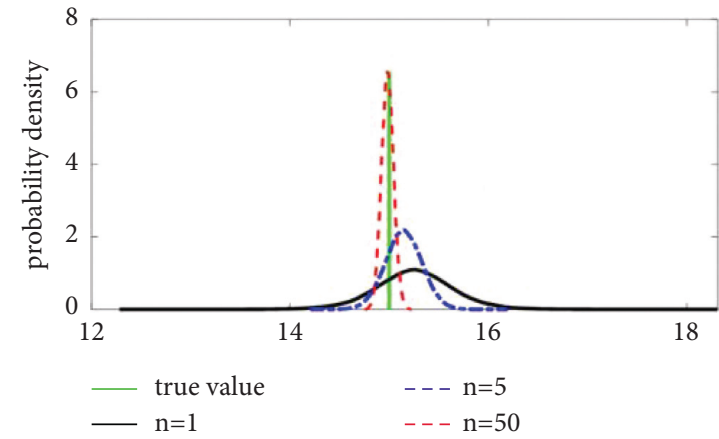

(a)

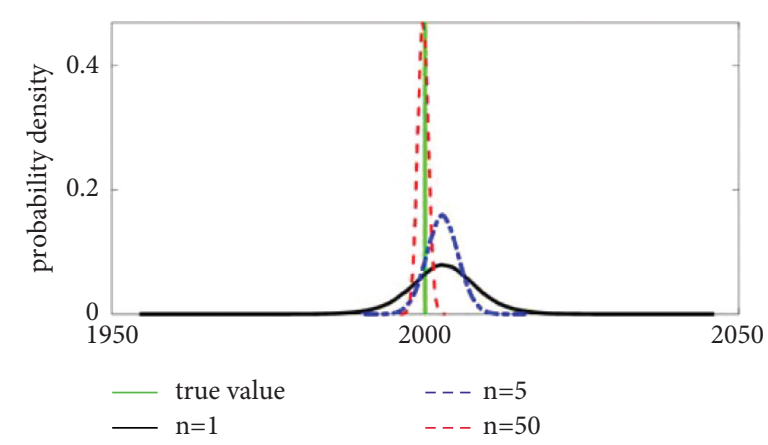

(c)

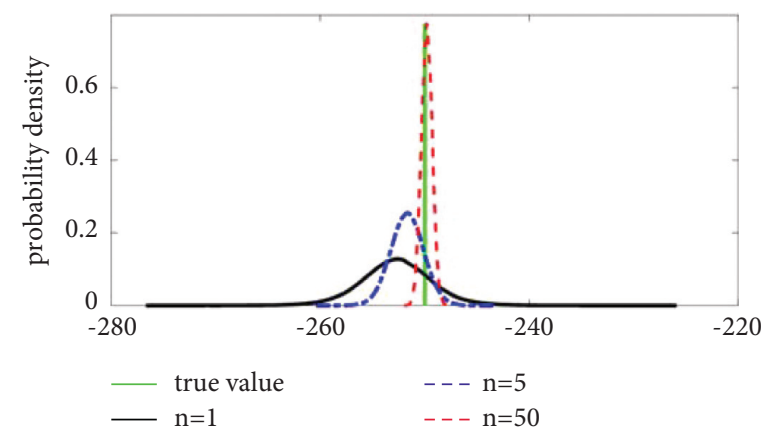

(b)

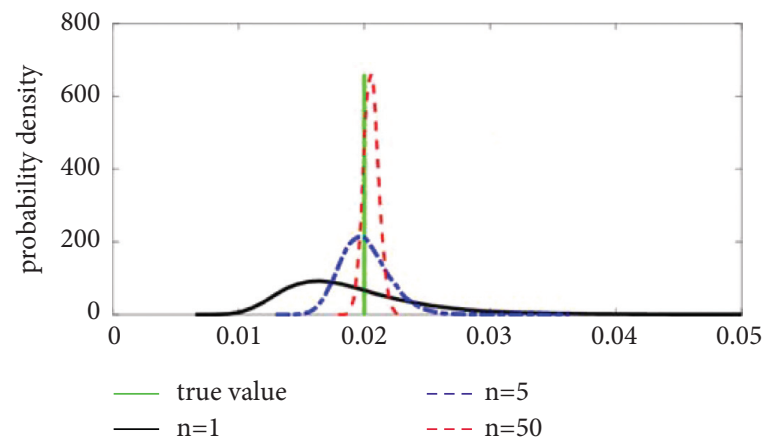

(d)

FIgURE 7: The posterior PDFs of the parameters according to experimental data with different sizes. 

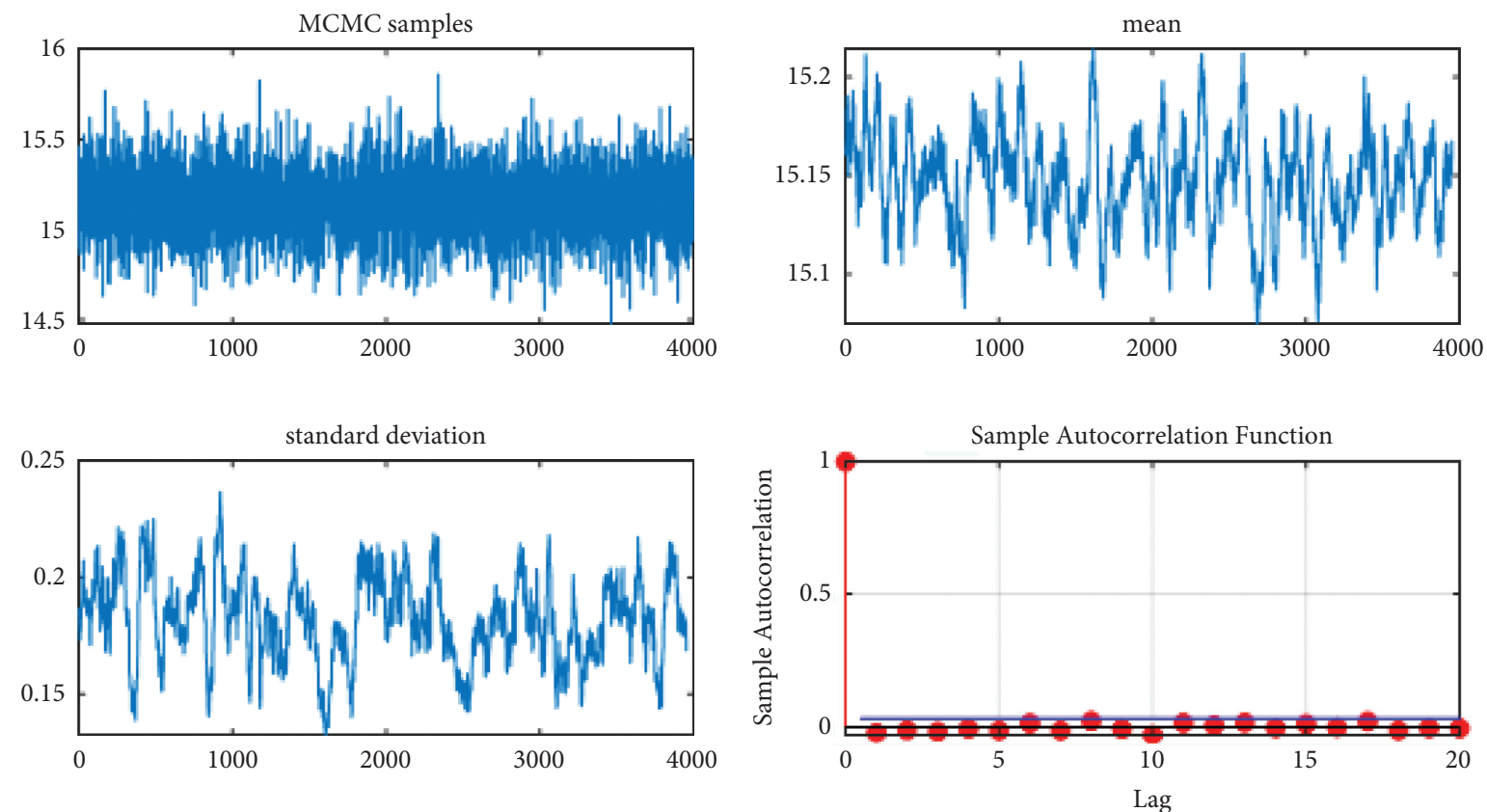

Figure 8: The sequence of MCMC samples for parameter $a$ to verify the convergence and autocorrelation.
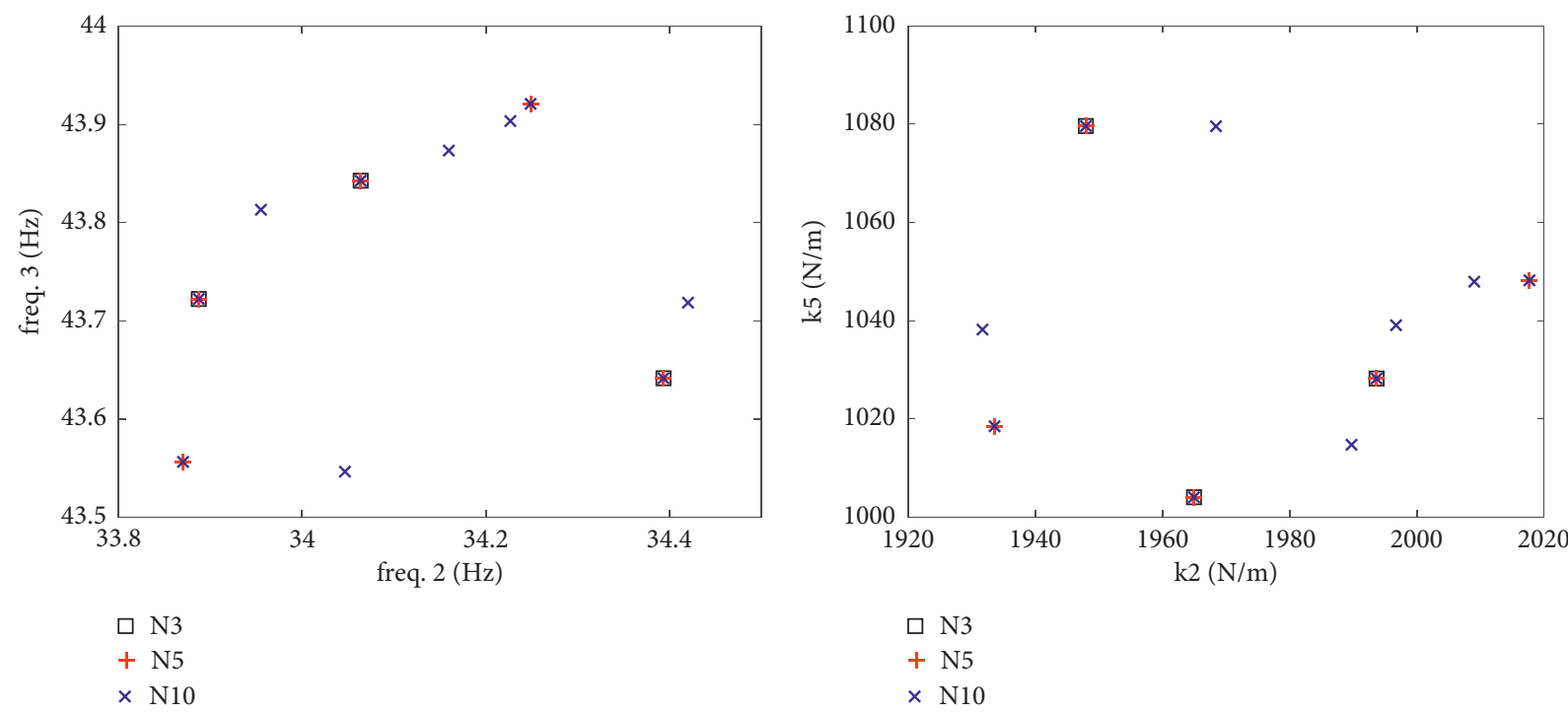

Figure 9: Virtual experimental frequency samples for case study 2 (a) and corresponding samples of spring stiffness (b).

the epistemic uncertainty of prior information is essentially reduced. Thus, the posterior PDFs for the hyperparameters become sharper and the highest probability estimation is closer to true value as shown in Figure 10 and Table 3. The probability distribution of the model parameter becomes closer to the true one as shown in Figure 11. Though the identification results become more accurate as the increase of the experimental sample size as shown in Figure 12, the influence of prior distribution seems much more obvious. It also reminds us that prior distribution should be carefully assigned. Improper sharp prior distributions may mislead the identification results.
It should be noted that the standard deviation of measurement noise is not identified in this case study. The author insists that measurement error should be obtained through instrument calibration as a known parameter rather than identified together with other parameters. In fact, it could confuse the identification results of aleatory uncertain parameters.

4.4. Case Study 3: Identification of Both Nonlinear and Linear Stiffness Together with Its Uncertainty. Both the nonlinear stiffness $\left(k_{4}\right)$ and linear stiffness $\left(k_{2}\right.$ and $\left.k_{5}\right)$ are identified in 

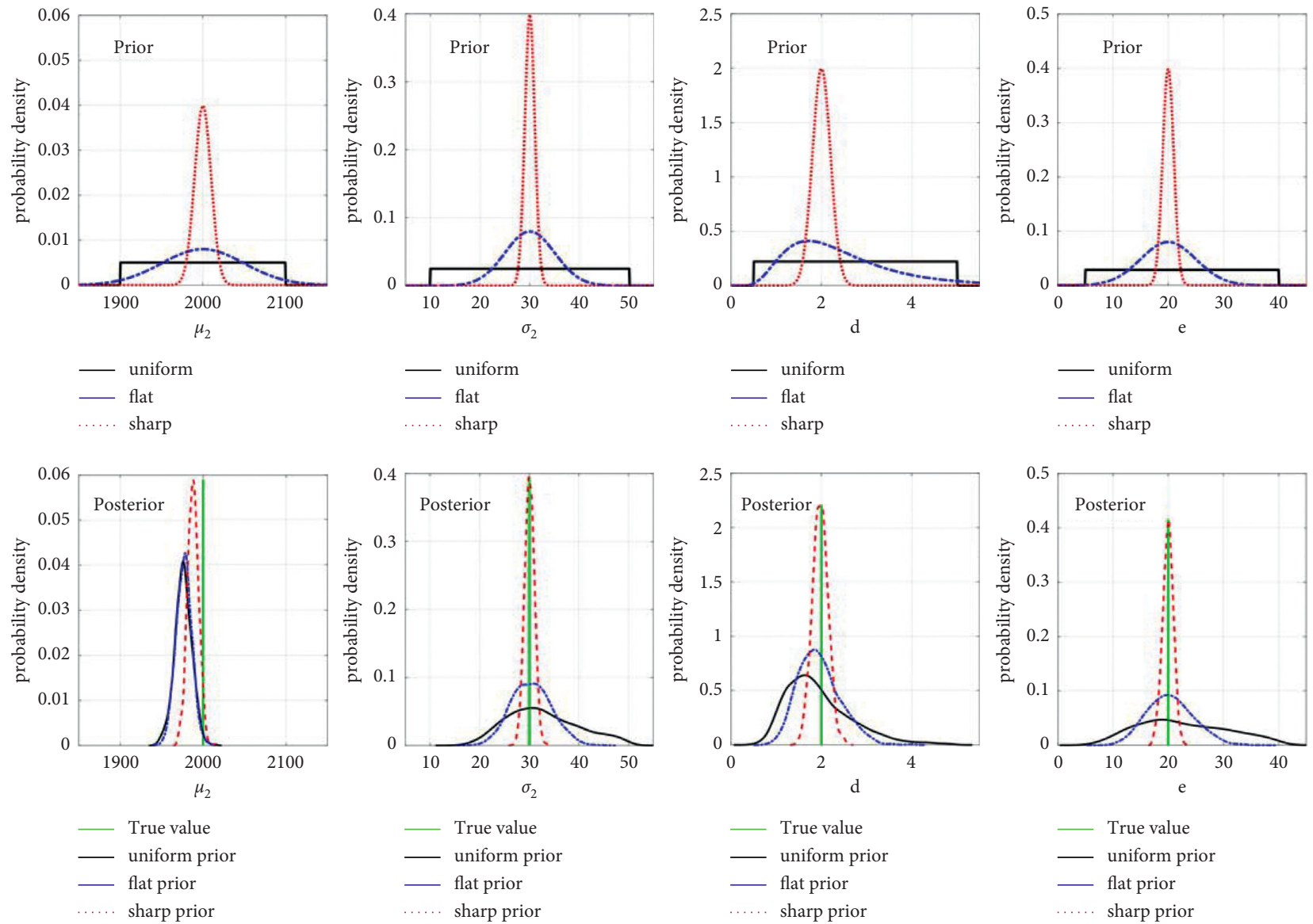

Figure 10: Comparison of the posterior PDFs for each hyperparameter according to different priors.
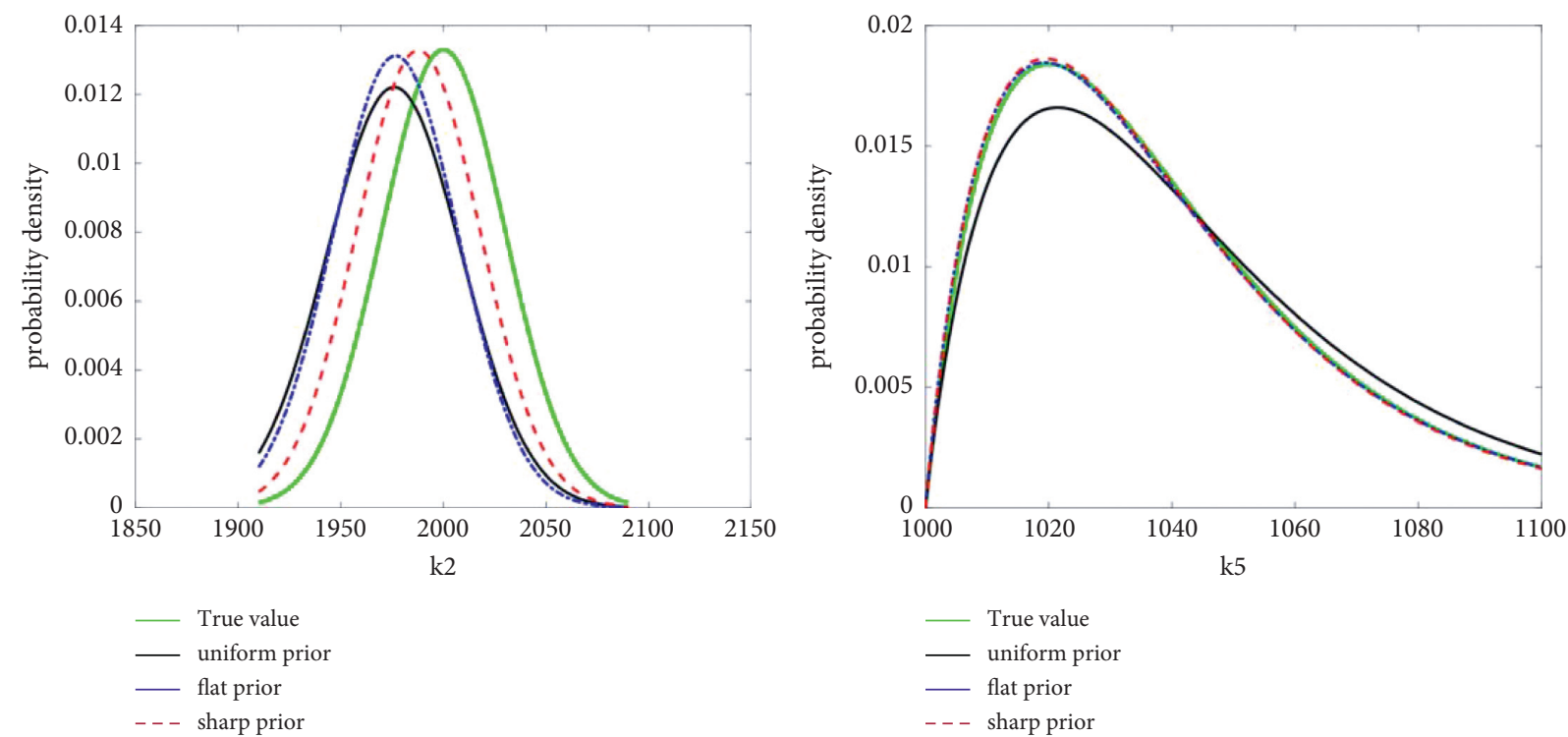

FIGURE 11: Identification results of PDFs for spring stiffness according to different priors.

this case study as well as their uncertainty. The constant term $c$ expressed in (20) is set as the normal distribution to address the aleatory uncertainty of nonlinear stiffness $k_{4}$. Virtual experimental samples for frequencies are generated in 20 groups under 6 different excitation magnitudes. The 20 groups of samples imply the aleatory uncertainty of parameters. The boxplots of test data and corresponding samples for aleatory uncertain parameters are shown in 
TABLE 3: Identification errors of the hyperparameters for aleatory uncertain variables.

\begin{tabular}{|c|c|c|c|c|c|}
\hline \multicolumn{2}{|c|}{ Subcase } & \multirow{2}{*}{$\frac{\mu_{2}(\%)}{-1.20}$} & \multirow{2}{*}{$\frac{\sigma_{2}(\%)}{8.93}$} & \multirow{2}{*}{$\frac{d(\%)}{-2.76}$} & \multirow{2}{*}{$\begin{array}{l}e(\%) \\
13.48\end{array}$} \\
\hline \multirow{3}{*}{ Different priors } & Uniform & & & & \\
\hline & Flat & -1.16 & 1.37 & -2.52 & 1.86 \\
\hline & Sharp & -0.61 & 0.19 & -1.18 & -0.22 \\
\hline \multirow{3}{*}{ Different sample sizes } & $n=10$ & -1.16 & 1.37 & -2.52 & 1.86 \\
\hline & $n=5$ & -1.21 & 2.27 & -12.66 & 2.00 \\
\hline & $n=3$ & -1.24 & -2.72 & -9.01 & 2.38 \\
\hline
\end{tabular}
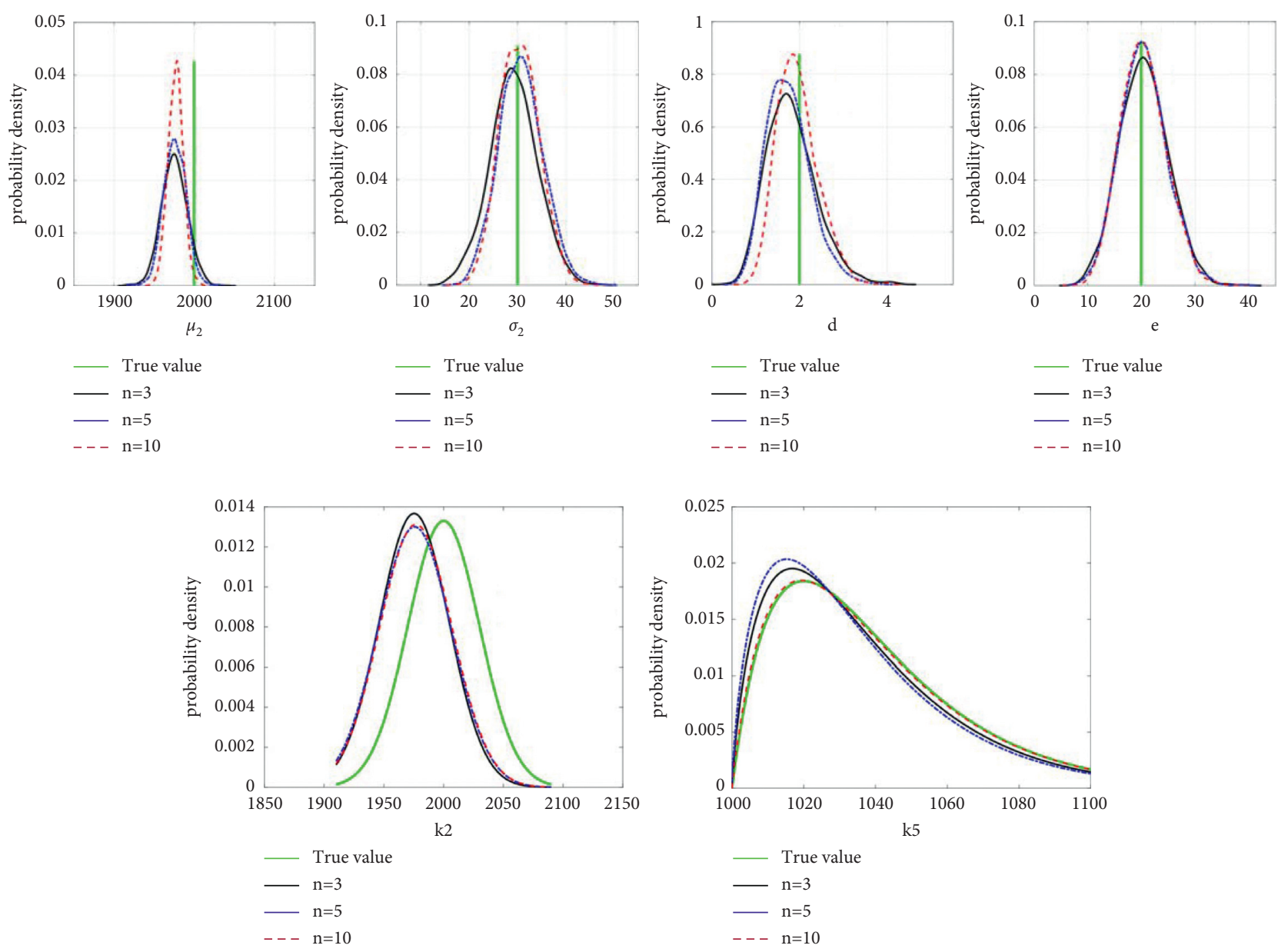

FIGURE 12: Influence comparison of experimental data amount on identification of aleatory uncertain parameter.

Figure 13. In this case study, the aleatory and epistemic uncertain parameter sets are $\theta=\left\{k_{2}, k_{5}, c\right\}$ and $\alpha=\{a, b\}$, respectively, while hyperparameter set is $\alpha_{2}=\left\{\mu_{2}, \sigma_{2}, d, e, \mu_{c}, \sigma_{c}\right\}$.

Adopting uniform prior distributions, the posterior PDFs of epistemic uncertain parameters and hyperparameters are shown in Figure 14. Both the posterior mean and highest posterior density estimation are, respectively, listed as the identification results shown in Table 4 . These two kinds of identification results show minor difference, while the latter is slightly more accurate. Since the former is more robust in general, the posterior mean is recommended as the optimal estimation in this paper.

According to every set of possible values for hyperparameters, plausible PDFs of spring stiffness are obtained as plotted with grey lines in Figure 15. According to the posterior mean of the hyperparameter, the optimal PDFs of spring stiffness are obtained as plotted with black lines to compare with true distribution plotted with green dotted lines in Figure 15. Furthermore, the expectation and 95\% confidence interval (CI) for $k_{4}$ in specified input could also be gained from the corresponding optimal PDF. The optimal expectation and bounds of $95 \%$ CI for $k_{4}$ varying with excitation magnitude are plotted with red solid and blue dashed lines, respectively, in Figure 15.

One of the important purposes of parameter identification is highlighted to improve the model accuracy and prediction capability. The natural frequencies of the system are predicted by the updated model as shown in Figure 16. The expectations varying with excitation magnitude are 

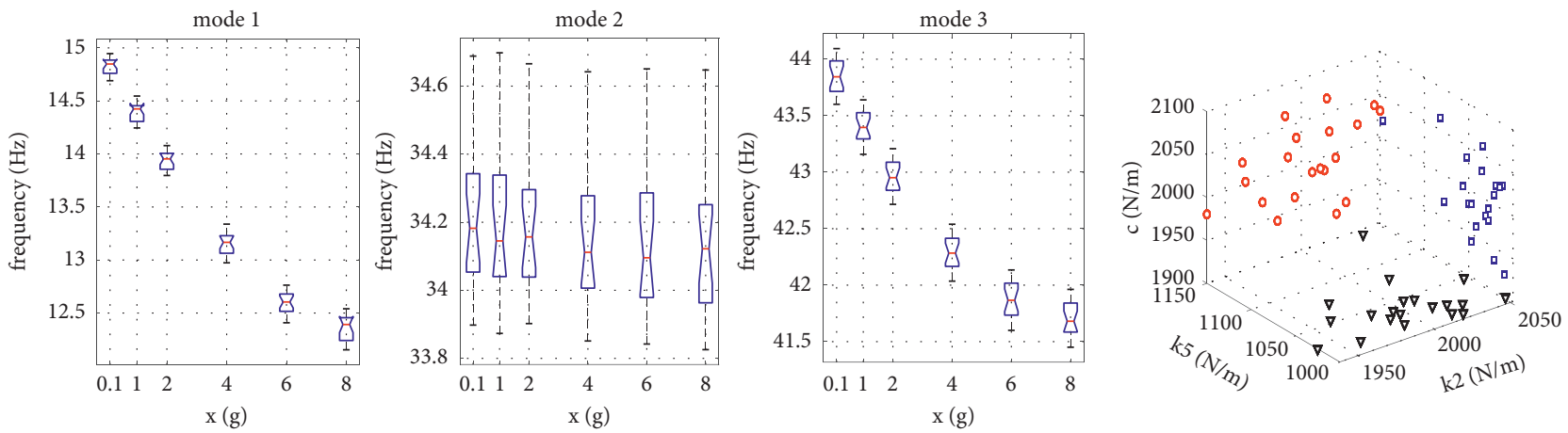

FIGURE 13: Boxplots of virtual frequency data for case study 3 and the projective plots of the corresponding 20 samples for aleatory uncertain parameters.
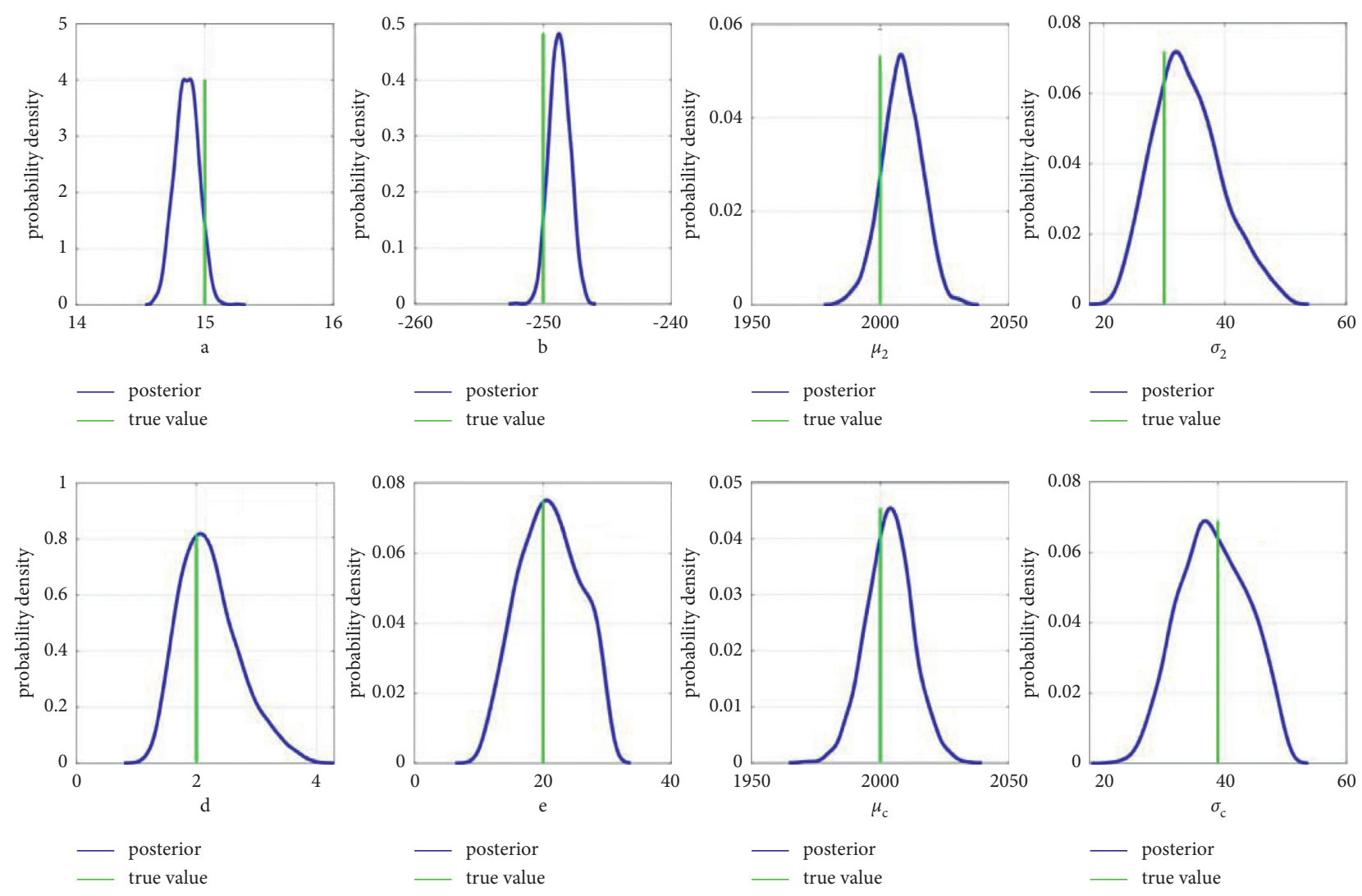

posterior
true value

posterior

FIGURE 14: Posterior PDFs of the epistemic uncertain parameters and hyperparameters.

TABLE 4: Identification errors of the epistemic uncertain parameters and hyperparameters.

\begin{tabular}{lccccc}
\hline \multirow{2}{*}{ Parameter } & True value & Palue & Resterior mean & \multicolumn{2}{c}{$\begin{array}{c}\text { Highest posterior density estimation } \\
\text { Value }\end{array}$} \\
\hline$a$ & 15 & 14.86 & -0.93 & 14.88 & -0.77 \\
$b$ & -250 & -248.74 & -0.51 & -248.79 & -0.48 \\
$\mu_{2}$ & 2000 & 2008.28 & 0.41 & 2007.79 & 0.39 \\
$\sigma_{2}$ & 30 & 33.93 & 13.10 & 31.90 & 2.07 \\
$d$ & 2 & 2.22 & 11.14 & 20.69 & 3.30 \\
$e$ & 20 & 21.05 & 0.17 & 2003.75 & 3.43 \\
$\mu_{c}$ & 2000 & 2003.44 & -1.60 & 38.00 & -4.99 \\
$\sigma_{c}$ & 40 & 39.36 & & & \\
\hline
\end{tabular}



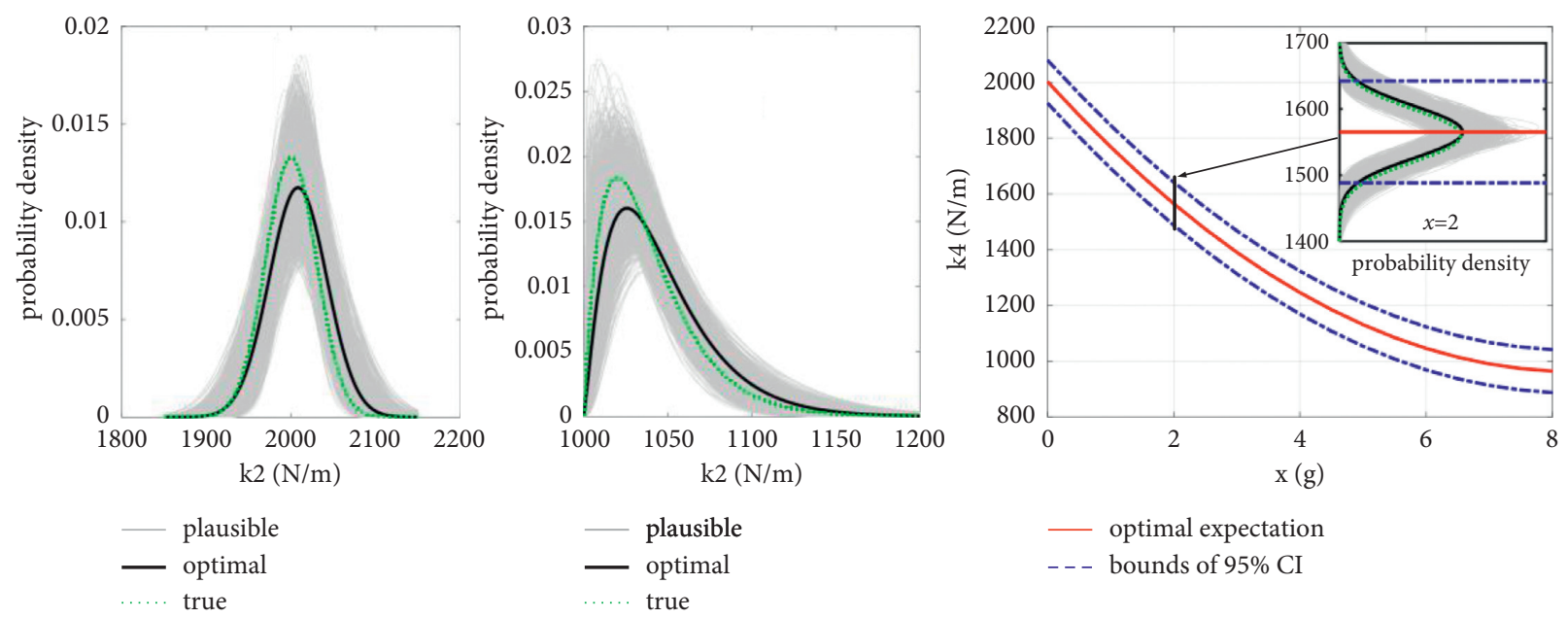

FIGURE 15: Identification results of PDFs for equivalent spring stiffness. Plausible PDFs are plotted with grey lines, and the optimal PDF is plotted with black lines.
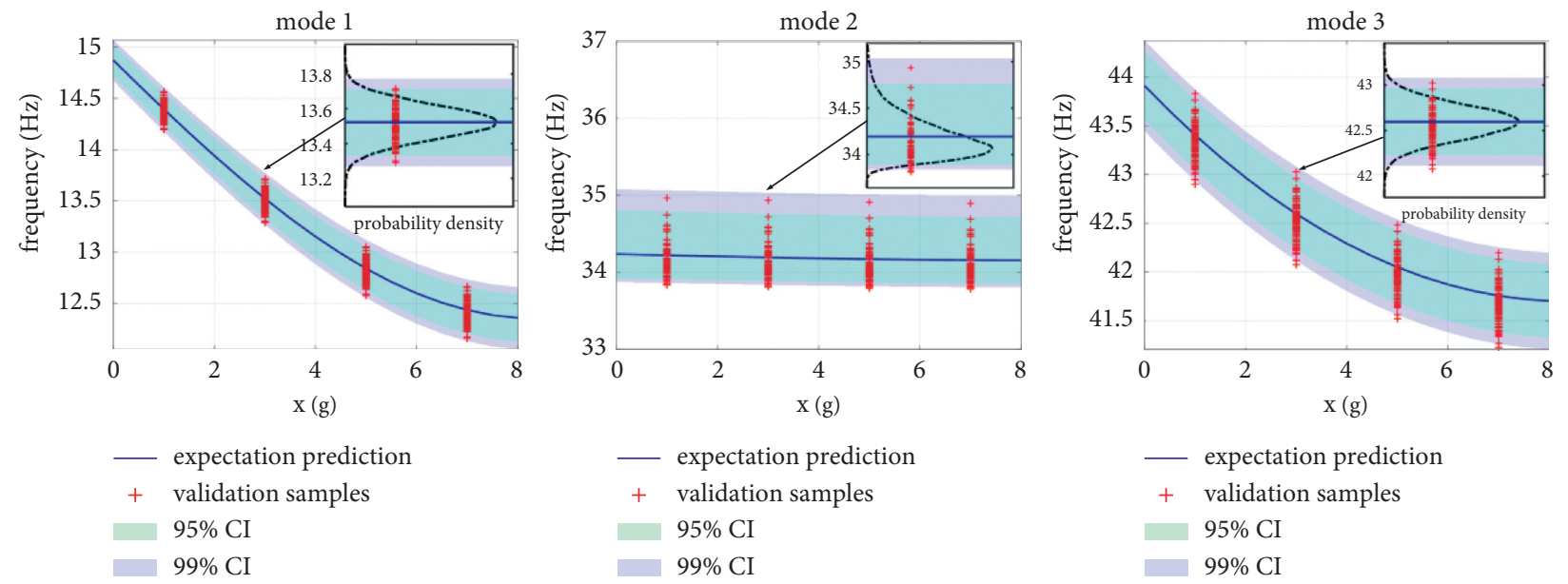

FIGURE 16: Prediction stripe with probability distribution for natural frequencies versus excitation magnitude.

plotted with blue solid lines. The 95\% and 99\% CIs are plotted with green and purple stripes. Moreover, the PDFs of frequencies at any specified excitation could be obtained as plotted with black dashed lines. It is demonstrated that shifting down of the $1^{\text {st }}$ and $3^{\text {rd }}$ frequencies are captured with the increase of excitation magnitude, and the uncertainties of the frequencies are also represented with PDFs or CIs for decision-making. By contrast, only optimal estimation plotted with red solid line in Figure 15 and expectation prediction plotted with blue solid line in Figure 16 could be accurately obtained in traditional Bayesian method.

Furthermore, 100 sets of experimental samples for frequencies at $x=1,3,5$ and 7 , respectively, are generated and marked with red "+" in Figure 16 to validate the prediction stripes. The result shows that the $95 \%$ CI covers most of the validation points and the $99 \% \mathrm{CI}$ covers almost all of them. It is indicated that the model updated by the proposed method has excellent predictive capability.

\section{Engineering Example}

The jointed structure suspended by elastics is randomly vibrated by a shaker at the bottom as shown in Figure 17. The thick disk and the cylindrical mass block are connected by four rubber isolators to reduce the vibration of the block, which represents some important elements to be protected. The modal tests are performed using 10 groups of different rubber isolators under random force excitation with 5 different levels. The experimental samples of axial natural frequency are plotted in Figure 18. It is indicated that the frequency shifts down obviously with the increase of excitation force. Moreover, the aleatory uncertainty of the isolator stiffness due to the notable difference of the rubber material is also significant and should be quantified.

A nonlinear probabilistic model of equivalent spring stiffness $k_{e}$ varying with excitation magnitude is established by first-order approximation, as shown in the following equation: 


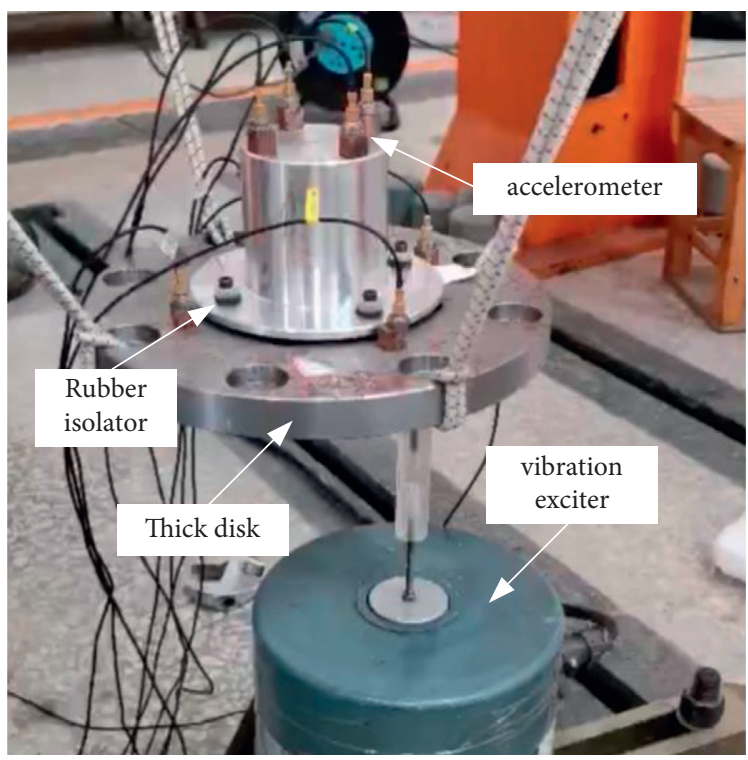

FIGURE 17: Experimental setup of the jointed structure.

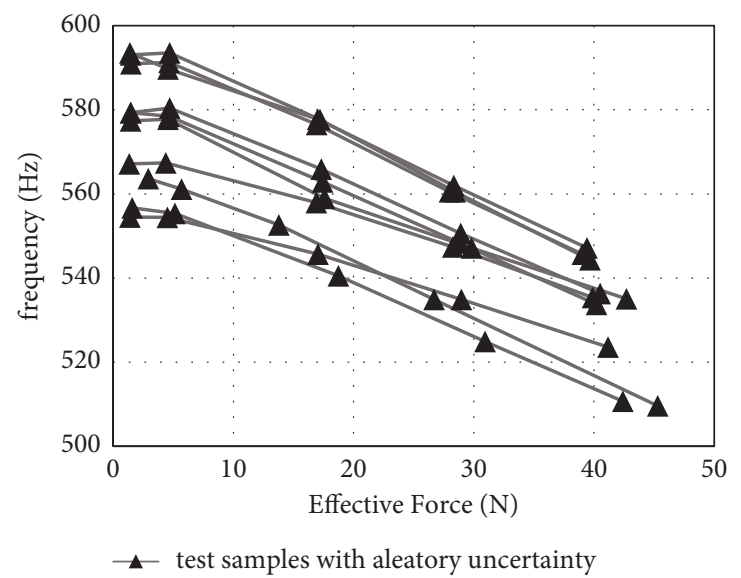

FIGURE 18: The natural frequency results from modal test using 10 groups of different isolators under 5 different excitation magnitudes.

$$
\left\{\begin{array}{l}
k_{e}=p F+k_{0} \\
k_{0} \sim N\left(\mu_{0}, \sigma_{0}^{2}\right)
\end{array}\right.
$$

where $F$ is the effective force of random excitation, $k_{0}$ is the underlying linear stiffness of the structure, and the parameter $p$ denotes the gradient of equivalent stiffness with excitation. $k_{0}$ is normal distributed with unknown hyperparameters $\mu_{0}$ and $\sigma_{0}$, and $p$ is the epistemic uncertain variable to be identified.

The finite element model of the jointed structures is established, and the rubber isolators are simulated by spring element with equivalent stiffness $k_{e}$. Polynomial response surface is also built up as the surrogate model for low computational cost. Thus, the model parameters, as well as their uncertainties, are identified by VEB method according to experimental frequency data. The posterior PDFs of uncertain model parameters are shown in Figure 19. Accordingly, the optimal estimations of the parameters are shown in Table 5 .
The prediction results including the expectation and $95 \%$ $\mathrm{CI}$ of the structural natural frequency are shown in Figure 20. It should be noted that the frequency expectation plotted with solid red line indicates the prediction of determinate nonlinear model, while the PDF at any specified force indicates the uncertainty prediction of the structural natural frequency.

Though the determinate nonlinear model could address the trend of frequency shift with effective force, the quantitative uncertainty of the frequency is unknown for decision-making. If the nonlinearity is not characterized in the model, the uncertainty range of the frequency would be very wide in order to cover all the test data. Thus, the uncertainty range may be useless for decision-making. However, combining the nonlinear prediction with the uncertainty, the 95\% CI shows excellent capability of prediction as shown in Figure 20 with light purple stripe. It completely covers the test samples and accurately describes the nonlinearity and 

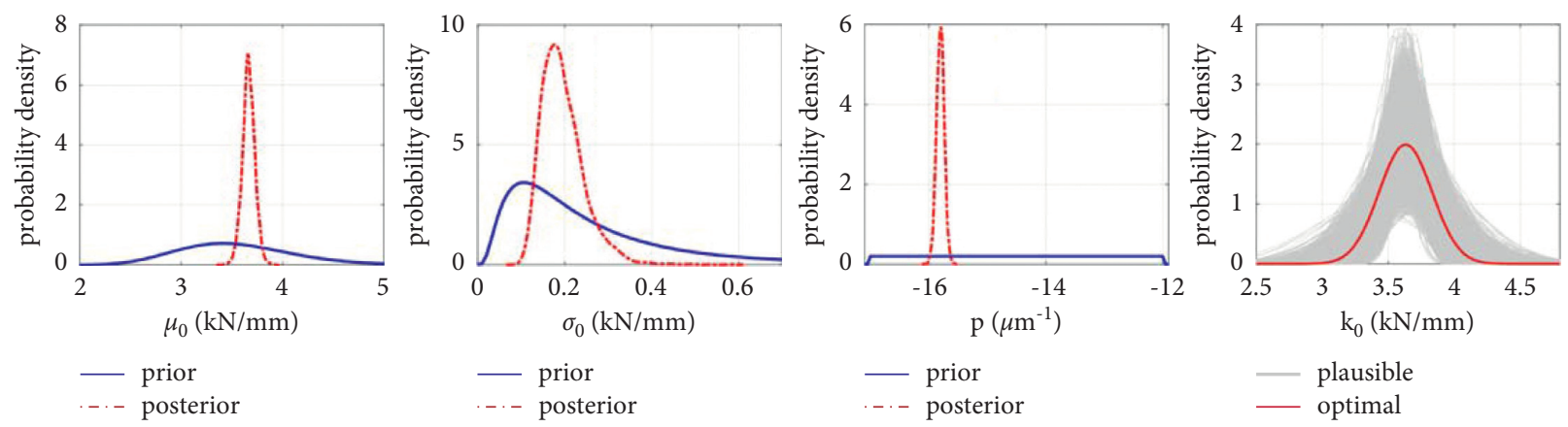

FiguRe 19: Identification results of PDFs for model parameters.

TABLE 5: Identification results for the engineering example.

\begin{tabular}{lc}
\hline Parameter & Optimal value \\
\hline$p\left(\mu \mathrm{m}^{-1}\right)$ & -15.804 \\
$\mu_{0}\left(\mathrm{kN} \cdot \mathrm{mm}^{-1}\right)$ & 3.6647 \\
$\sigma_{0}\left(\mathrm{kN} \cdot \mathrm{mm}^{-1}\right)$ & 0.1957 \\
\hline
\end{tabular}

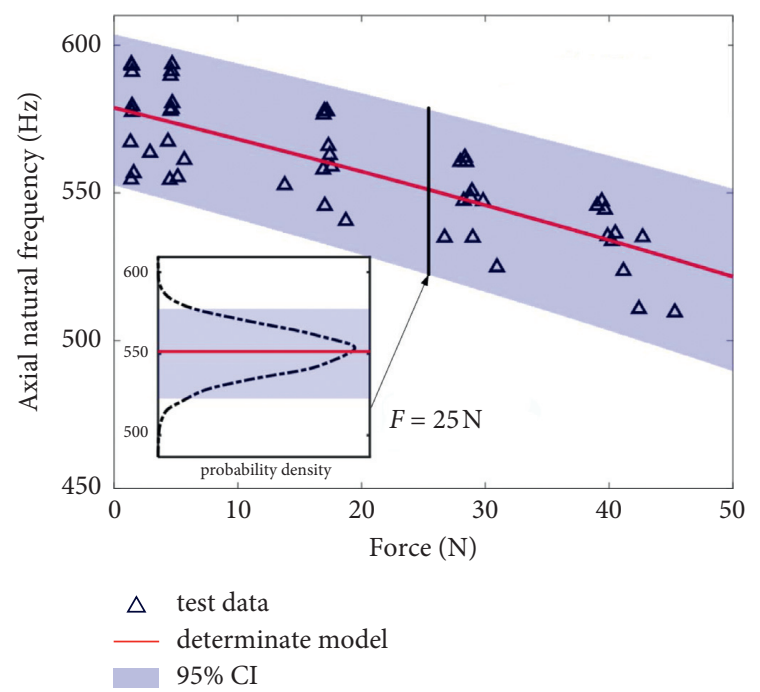

Figure 20: Prediction of the frequency with nonlinearity and uncertainty.

uncertainty, which demonstrates significant practicability for engineering structures.

\section{Conclusions}

In order to address both the nonlinearity and uncertainties of jointed structures under random excitation, equivalent linearization and Bayesian identification method are used in this paper. A novel method of VEB identification is originally proposed to obtain the optimal estimation and the probability distribution of model parameters, especially for the parameters with aleatory uncertainty. The numerical example with 3 case studies is performed to illustrate and verify the proposed method. It is demonstrated that the model parameters identified by VEB method are very close to their true values, and they would be much closer as the supplement of more experimental data or more accurate priors. The engineering example of the jointed structure with rubber isolators is also carried out to show the practicability and prediction capability. The nonlinear stiffness varying with the inputs is well identified together with its uncertainty in terms of PDF. Moreover, the outputs are also accurately predicted with the uncertain stripes varying with the inputs. It is indicated that both the nonlinear feature and the uncertainties of the output are characterized to support decision-making in our method, which has significant practicability for analysis of engineering structures. The limitation of this method also shows that the convergence rate of MCMC would obviously slow down with the increasing of the number of model parameters. As a result, the identification should be avoided for high-dimensional (i.e., more than 20) parameters especially with aleatory uncertainties.

\section{Data Availability}

The data used to support of this study are available from the corresponding author upon request.

\section{Conflicts of Interest}

On behalf of all authors, the corresponding author states that there are no conflicts of interest.

\section{Acknowledgments}

The authors acknowledge the funding supports from the National Natural Science Foundation of China (no. 11702280), the National Natural Science Foundation of China and National Safety Academic Foundation of China (,o. U1730129), and Innovation and Development Foundation of CAEP (no. CX2019014).

\section{References}

[1] M. R. W. Brake, The Mechanics of Jointed Structures, Springer International Publishing AG, Gewerbestrasse, Switzerland, 2018.

[2] S. Bograd, P. Reuss, A. Schmidt, L. Gaul, and M. Mayer, "Modeling the dynamics of mechanical joints," Mechanical Systems and Signal Processing, vol. 25, no. 8, pp. 2801-2826, 2011.

[3] G. Kerschen, K. Worden, A. F. Vakakis, and J.-C. Golinval, "Past, present and future of nonlinear system identification in 
structural dynamics," Mechanical Systems and Signal Processing, vol. 20, no. 3, pp. 505-592, 2006.

[4] T. Friis, M. Tarpø, E. I. Katsanos, and R. Brincker, "Equivalent linear systems of nonlinear systems," Journal of Sound and Vibration, vol. 469, no. 1, Article ID 115126, 2020.

[5] S. H. Crandall, "A half-century of stochastic equivalent linearization," Structural Control and Health Monitoring, vol. 13, no. 1, pp. 27-40, 2006.

[6] U. Alibrandi and K. M. Mosalam, "Equivalent linearization methods for stochastic dynamic analysis using linear response surfaces," Journal of Engineering Mechanics, vol. 143, no. 8, Article ID 4017055, 2017.

[7] J. M. Jara, G. Raya, B. A. Olmos, and G. Martinez, "Applicability of equivalent linearization methods to irregular isolated bridges," Engineering Structures, vol. 141, no. 1, pp. 495-511, 2017.

[8] Z. Zheng and H. Dai, "A new fractional equivalent linearization method for nonlinear stochastic dynamic analysis," Nonlinear Dynamics, vol. 91, no. 2, pp. 1075-1084, 2018.

[9] X. Chen, Z. Shen, Q. He, Q. Du, and X. Liu, "Influence of uncertainty and excitation amplitude on the vibration characteristics of rubber isolators," Journal of Sound and Vibration, vol. 377, no. 1, pp. 216-225, 2016.

[10] D. J. Ewins, "Exciting vibrations: the role of testing in an era of supercomputers and uncertainties," Meccanica, vol. 51, no. 11, pp. 1-20, 2016.

[11] C. Jiang, J. Zheng, and X. Han, "Probability-interval hybrid uncertainty analysis for structures with both aleatory and epistemic uncertainties: a review," Structural and Multidisciplinary Optimization, vol. 57, no. 6, pp. 2485-2502, 2018.

[12] J. C. Helton and J. D. Johnson, "Quantification of margins and uncertainties: a," Reliability Engineering \& System Safety, vol. 96, no. 9, pp. 1034-1052, 2011.

[13] I. Park and R. V. Grandhi, "A Bayesian statistical method for quantifying model form uncertainty and two model combination methods," Reliability Engineering \& System Safety, vol. 129, no. 1, pp. 46-56, 2014.

[14] Y.-C. Ni and F.-L. Zhang, "Uncertainty quantification in fast Bayesian modal identification using forced vibration data considering the ambient effect," Mechanical Systems and Signal Processing, vol. 148, Article ID 107078, 2021.

[15] D.-D. Tan, Z.-Q. Lu, D.-H. Gu, H. Ding, and L.-Q. Chen, "A ring vibration isolator enhanced by a nonlinear energy sink," Journal of Sound and Vibration, vol. 508, no. 1, Article ID 116201, 2021.

[16] Z.-Q. Lu, D.-H. Gu, H. Ding, W. Lacarbonara, and L.-Q. Chen, "Nonlinear vibration isolation via a circular ring," Mechanical Systems and Signal Processing, vol. 136, no. 1, Article ID 106490, 2020.

[17] Z. Lu, L.-Q. Chen, M. J. Brennan, T. Yang, H. Ding, and Z. Liu, "Stochastic resonance in a nonlinear mechanical vibration isolation system," Journal of Sound and Vibration, vol. 370, no. 1, pp. 221-229, 2016.

[18] J. E. Mottershead and M. I. Friswell, "Model updating in structural dynamics: a survey," Journal of Sound and Vibration, vol. 167, no. 2, pp. 347-375, 1993.

[19] G. Kerschen and J. Golinval, "Generation of accurate finite element models of nonlinear systems application to an aeroplane-like structure," Nonlinear Dynamics, vol. 39, no. 12, pp. 129-142, 2005.

[20] J. L. Beck and L. S. Katafygiotis, "Updating models and their uncertainties. I: bayesian statistical framework," Journal of Engineering Mechanics, vol. 124, no. 4, pp. 455-461, 1998.
[21] S. Kwag and B. S. Ju, "Application of a Bayesian hierarchical model to system identification of structural parameters," Engineering with Computers, vol. 36, no. 2, pp. 455-474, 2020.

[22] R. Rocchetta, M. Broggi, Q. Huchet, and E. Patelli, "On-line Bayesian model updating for structural health monitoring," Mechanical Systems and Signal Processing, vol. 103, no. 1, pp. 174-195, 2018.

[23] R. Astroza, N. Barrientos, Y. Li, E. I. Saavedra Flores, and Z. Liu, "Bayesian updating of complex nonlinear FE models with high-dimensional parameter space using heterogeneous measurements and a batch-recursive approach," Engineering Structures, vol. 201, no. 1, Article ID 109724, 2019.

[24] Y. Xin, H. Hao, J. Li, Z.-C. Wang, H.-P. Wan, and W.-X. Ren, "Bayesian based nonlinear model updating using instantaneous characteristics of structural dynamic responses," Engineering Structures, vol. 183, no. 1, pp. 459-474, 2019.

[25] I. Behmanesh, B. Moaveni, G. Lombaert, and C. Papadimitriou, "Hierarchical Bayesian model updating for structural identification," Mechanical Systems and Signal Processing, vol. 64-65, no. 1, pp. 360-376, 2015.

[26] H. Sun and R. Betti, "A hybrid optimization algorithm with bayesian inference for probabilistic model updating," Computer-Aided Civil and Infrastructure Engineering, vol. 30, no. 8, pp. 602-619, 2015.

[27] C. Soize, "Bayesian posteriors of uncertainty quantification in computational structural dynamics for low-and mediumfrequency ranges," Computers \& Structures, vol. 126, no. 1, pp. 41-55, 2013.

[28] K. Worden and G. R. Tomlinson, Nonlinearity in Structural Dynamics, Detection, Identification and Modelling, Institute of Physics Publishing, Bristol, England, 2001.

[29] P. L. Green and K. Worden, "Bayesian and Markov chain Monte Carlo methods for identifying nonlinear systems in the presence of uncertainty," Philosophical Transactions of the Royal Society A: Mathematical, Physical \& Engineering Sciences, vol. 373, no. 2051, Article ID 20140405, 2015.

[30] S. Ross, Stochastic Processes, John Wiley \& Sons, Hoboken, NJ, USA, 1996.

[31] J. Ching and Y.-C. Chen, "Transitional Markov chain Monte Carlo method for Bayesian model updating, model class selection, and model averaging," Journal of Engineering $\mathrm{Me}$ chanics, vol. 133, no. 7, pp. 816-832, 2007. 